The article Evaporating Foam Films of Pure Liquid Stabilized via the Thermal Marangoni Effect by Javor K. Novev, Nikolay Panchev and Radomir I. Slavchov has been published in the journal Chemical Engineering Science (DOI:10.1016/j.ces.2017.06.016) and is available online at http://www.sciencedirect.com/science/article/pii/S0009250917304050?via\%3Dihub.

(C) 2017. This manuscript version is made available under the CC-BY-NC-ND 4.0 license http://creativecommons.org/licenses/by-nc-nd/4.0/ 


\section{Accepted Manuscript}

Evaporating Foam Films of Pure Liquid Stabilized via the Thermal Marangoni Effect

Javor K. Novev, Nikolay Panchev, Radomir I. Slavchov

PII:

S0009-2509(17)30405-0

DOI: http://dx.doi.org/10.1016/j.ces.2017.06.016

Reference: CES 13660

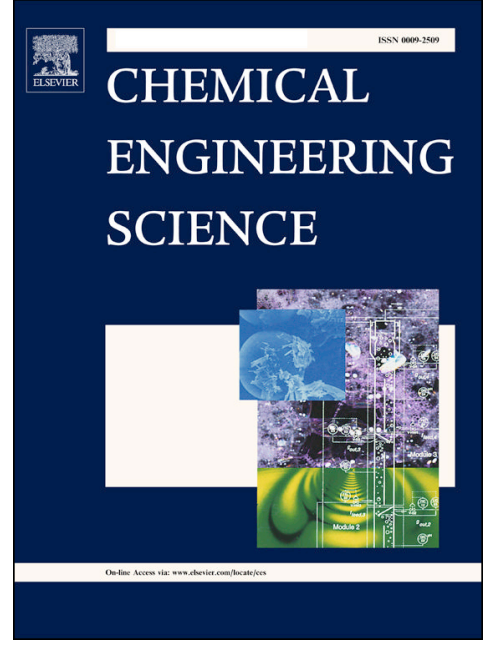

To appear in: $\quad$ Chemical Engineering Science

Received Date: $\quad 7$ March 2017

Revised Date: $\quad 6$ June 2017

Accepted Date: $\quad 7$ June 2017

Please cite this article as: J.K. Novev, N. Panchev, R.I. Slavchov, Evaporating Foam Films of Pure Liquid Stabilized via the Thermal Marangoni Effect, Chemical Engineering Science (2017), doi: http://dx.doi.org/10.1016/j.ces. 2017.06.016

This is a PDF file of an unedited manuscript that has been accepted for publication. As a service to our customers we are providing this early version of the manuscript. The manuscript will undergo copyediting, typesetting, and review of the resulting proof before it is published in its final form. Please note that during the production process errors may be discovered which could affect the content, and all legal disclaimers that apply to the journal pertain. 


\title{
Evaporating Foam Films of Pure Liquid Stabilized via the Thermal Marangoni Effect
}

\author{
Javor K. Novev ${ }^{1}$, Nikolay Panchev², Radomir I. Slavchov ${ }^{3}$ * \\ ${ }^{l}$ Department of Chemistry, Physical and Theoretical Chemistry Laboratory, University of \\ Oxford, Oxford OX1 3QZ, UK \\ ${ }^{2}$ Institute of Physical Chemistry, Bulgarian Academy of Sciences, Sofia 1113, Bulgaria \\ ${ }^{3}$ Department of Chemical Engineering and Biotechnology, University of Cambridge, Cambridge \\ CB3 OSA, UK \\ * Corresponding author (ris26@cam.ac.uk)
}

\begin{abstract}
A foam film made of pure liquid can be stabilized by evaporation. This is demonstrated experimentally for water and alkane films formed in a Scheludko cell at controlled saturation of the ambient air. A mechanism of the stabilization is proposed: evaporation leads to a local decrease of the temperature in the centre of the film; the meniscus acts as a thermostat and maintains a higher temperature at the film periphery. The resulting temperature gradient brings about a surface tension gradient causing a stabilizing thermal Marangoni flow that carries fluid from the meniscus to the interior of the film. The film thickness is quasi-stationary and gradually decreases as the meniscus cools due to the evaporation. At a certain critical meniscus temperature, the film reaches a critical thickness at which the Marangoni effect can no longer counteract the combined action of the capillary pressure and the van der Waals attraction, and the film breaks. The lifetime of the film is estimated as a function of the film size and the experimental conditions (temperature, saturation, vapour pressure, capillary pressure). The theoretical and the experimental results for the lifetime and the critical thickness are in qualitative agreement for films at moderate saturation.
\end{abstract}

Keywords: Marangoni effect; evaporation; foam film stability; surfactant-free films; Scheludko cell; alkane films. 


\section{Introduction}

The drainage and stability of thin liquid films determine the properties of a broad range of objects, including foam, bakery yeast (Exerowa and Kruglyakov, 1998), cement (Du and Folliard, 2005), water-crude oil emulsions (Sullivan and Kilpatrick, 2002), and many others. The process of evaporation is known to influence the stability of these films. In particular, there are numerous studies on evaporating wetting films on solid substrates due to their importance for certain technological processes, such as fibre optic coating, semiconductor chip deposition (Danov et al., 1998), fuel atomization in engines (Baumgarten, 2006), etc. Evaporation interferes with these processes primarily by causing Marangoni flows (Baumgarten, 2006; Danov et al., 1998; Sultan et al., 2005; Yiantsios and Higgins, 2006; Zuiderweg and Harmens, 1958). In contrast, the research on evaporating foam films is quite limited (Manev and Nguyen, 2005) and to our knowledge, only a handful of studies devote attention to evaporating thin films containing no surfactant, e.g. (Yaminsky et al., 2010a). The related problem for evaporation of sessile drops from pure liquids has been studied in greater depth (Chandramohan et al., 2016; Hu and Larson, 2006, 2005).

Evaporation has a twofold effect on the stability of thin films - by taking away heat and mass from the film, it brings about thermal Marangoni flows (driven by a temperature gradient) and Gibbs-Marangoni flows (driven by concentration gradients in multicomponent liquids). In the case of a wetting film, the solid substrate acts as a thermostat and reduces the magnitude of the thermal Marangoni effect. Due to the absence of this thermostating effect, it can be expected that the Marangoni flow is stronger in evaporating foam films. There are hints in the literature in support of this hypothesis. For example, Li et al. (Li et al., 2012) reported that bubbles formed from surfactant solutions burst under non-uniform evaporation. In their experiment, evaporation leads to a local thinning of the liquid films, as well as to a local increase of the surfactant concentration, resulting in a gradient of the surface tension $\sigma$. This gradient causes a Marangoni flux away from the thinner region of more intense evaporation and leads to the bursting of the bubble. In contrast, Manev et al. (Manev, 1975; Manev and Nguyen, 2005) studied evaporating foam films formed from surfactant solutions in a Scheludko cell and found no significant effect of evaporation in these systems. Yaminsky et al. (Yaminsky, 2006; Yaminsky et al., 2010a, 2010b) hypothesized that evaporation-driven Marangoni effects are the cause of the stabilization 
of the foam films they observed in a variety of experimental setups. Their surfactant-free films reacted particularly strongly to evaporation. Yaminsky (Yaminsky, 2006) reported that when a small air bubble attaches to the interface between air and a pure liquid containing no surfactant, its behaviour is dependent upon the saturation - if the air is saturated with vapour, the bubble coalesces immediately. Conversely, in an unsaturated environment, the air|liquid|air film formed upon contact between the bubble and the surface remains stable over an indefinite period of time. Another interesting phenomenon occurring in evaporating films free of surfactant was reported by Karakashev and co-authors (Karakashev et al., 2008). Contrary to expectations based on the Derjaguin-Landau-Verwey-Overbeek theory, their foam films exhibited significant stability (lifetime $\sim 100 \mathrm{~s}$ ) and a complex dynamic behaviour despite being formed from concentrated solutions of inorganic electrolytes. This anomalous stability was only displayed when the surrounding gas was undersaturated with respect to the vapour pressure of the salt solution (Karakashev et al., 2008).

These reports suggest that evaporation plays a key role in the stabilization of surfactant-free foam films against the capillary pressure-driven drainage and generated our interest in exploring this topic. The salt films studied by Karakashev et al. (Karakashev et al., 2008) are complex systems - the observed intense fluxes must be affected by the evaporation-induced gradient of the electrolyte concentration and the effect of the electrolyte on surface tension (Slavchov and Novev, 2012). Evidently, evaporation-driven Gibbs-Marangoni flows are also present in surfactant solutions; for this reason, trace amounts of surfactant have a destabilizing effect on evaporating films of pure liquid (Yaminsky et al., 2010a). Instead of tackling the complicated problem of two-component films, in this study we explore the simpler case of an evaporating film comprised of pure liquid, where only the thermal Marangoni effect is present.

Even in this case, however, the behaviour of the film is influenced by many factors other than the relative saturation, complicating the interpretation of the experimental data. There is no consensus in the literature about the stability of pure water foam films even in the absence of evaporation (Peng and Chang, 2014): while some authors state that they rupture instantly and even use this as a criterion for the purity of their experimental setup (Karakashev et al., 2008; Nguyen and Nguyen, 2010), others report stable films of this kind (Exerowa, 1969; Exerowa and Kruglyakov, 1998; Yaminsky et al., 2010a). The reported lifetime of such films varies by orders of magnitude depending on the method used for their preparation. Karakashev and Firouzi 
(Karakashev and Firouzi, 2014) observed stable water films; however, they became unstable following a 200-fold flushing of the entire cell with water. In a saturated environment, after the flushing, pure water films were found to have a lifetime of $\sim 5 \mathrm{~s}$, reaching a thickness of $\sim 30 \mathrm{~nm}$ before rupture. In an undersaturated environment, such films ruptured instantly.

Several studies report that the stability of pure water films is also dependent upon the approach velocity of the two water|air interfaces (Del Castillo et al., 2011; Firouzi and Nguyen, 2014; Wang and Qu, 2012; Yaminsky, 2006; Yaminsky et al., 2010a, 2010b). In a saturated environment, if the velocity is above a certain critical value $(35 \mu \mathrm{m} / \mathrm{s}$ (Firouzi and Nguyen, 2014), $200 \mu \mathrm{m} / \mathrm{s}$ (Yaminsky et al., 2010b) or less than $11 \mu \mathrm{m} / \mathrm{s}$ (Wang and Qu, 2012)), the two surfaces coalesce instantly. Firouzi and Nguyen (Firouzi and Nguyen, 2014) found that films live for several seconds at an approach velocity of under $35 \mu \mathrm{m} / \mathrm{s}$. Yaminsky et al. reported a similar finding at both saturated (Yaminsky et al., 2010b) and undersaturated (Yaminsky et al., 2010a) conditions: for approach velocities between $\sim 1$ and $\sim 100 \mu \mathrm{m} / \mathrm{s}$, the average lifetime of their films was about a minute, while below $1 \mu \mathrm{m} / \mathrm{s}$, it was of the order of hours. Numerous studies devoted to the coalescence of gas bubbles with the water|air interface show a similar dependence on the approach velocity (Del Castillo et al., 2011; Katsir and Marmur, 2014a, 2014b). The stability at low approach rates is attributed to electrostatic repulsion between the two interfaces (Katsir and Marmur, 2014b; Yaminsky et al., 2010b). It is not entirely clear what the cause for this electrostatic repulsion is or by what mechanism it is overcome at high approach rates. Finally, a persistent reason for contradicting reports is the presence of surface-active impurities. Such impurities generally stabilize films under saturated conditions; however, evaporating films made of pure liquid can in fact be destabilized by trace quantities of surfactants (Yaminsky et al., 2010a).

The short review above demonstrates that, though it may not be entirely consistent, there is a body of evidence indicating that films formed from single-component liquids may be stabilized by evaporation. Our aim in this study is to clarify the question by (i) considering the possible mechanism of stabilization theoretically and (ii) investigating evaporating films experimentally under controlled saturation of the ambient air. The basic hypothesis we will test is that evaporation gives rise to a thermal Marangoni effect - an idea formulated by Yaminski et al. (Yaminsky et al., 2010a). The model we will present in Section 2 generalizes the classical Maxwell-Langmuir-Fuchs theory (Fuchs, 1959) of the evaporation of a spherical droplet to an 
approximate geometry mimicking the biconcave droplet in the Scheludko cell (Sheludko, 1967); it can also be viewed as a thermal-Marangoni-effect analogue of Marrucci's theory of the stabilization of films by the Gibbs-Marangoni effect (Marrucci, 1969). We use this model to analyse the behaviour of several measurable quantities with respect to changes in the experimentally controllable parameters - particularly interesting are: the evolution of the film thickness; the film lifetime as a function of its size and the ambient vapour pressure; the critical parameters of film rupture - film thickness, radius, saturation - as a function of the properties of the liquid. For the purpose of testing the qualitative predictions of this model, we performed experiments with evaporating films made of pure water and alkanes under controlled saturation of the ambient air. The results are discussed in Section 3.

\section{A model for the stability of an evaporating film}

The model we develop considers a disc-shaped foam film in contact with a ring-like meniscus. This mimics the common experimental setup for studying foam films - the Scheludko cell (Sheludko, 1967), Figure 1A, where the film is formed by withdrawing liquid from a biconcave drop situated in a glass capillary (film holder). The typical dimensions in this setup are $R_{\mathrm{c}} \sim 2$ $\mathrm{mm}$ for the radius of the capillary, $R_{\mathrm{f}} \sim 150 \mu \mathrm{m}$ for the film radius and $h \sim 100 \mathrm{~nm}$ for the film thickness (for the reader's convenience, all symbols and parameter values are tabulated in the supplement S1). The capillary is at a fixed position inside a container filled with air of controlled saturation, Figure 1B; the container is a cylinder of radius $R_{\text {cont }} \sim 1 \mathrm{~cm}$ and height $L_{\text {cont }} \sim 9 \mathrm{~cm}$.

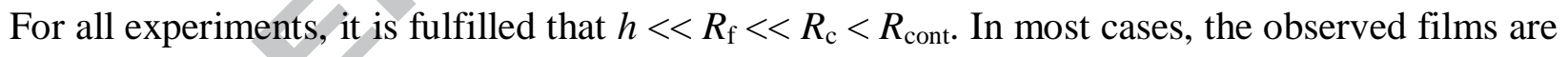
approximately plane-parallel - the thickness of the film zone is homogeneous, resulting in homogeneous colour, Figure 1C.

The evaporation gives rise to several conjugated processes in this system:

(i) as evaporation is endothermic, it causes a local temperature drop to develop within the thin film. The temperature of the meniscus is affected to a lesser degree because both the relatively large amount of liquid in it and the glass wall that is in contact with it exert a thermostating effect. This produces a radial temperature gradient, $\nabla T$, in the film. 
(ii) The temperature gradient $\nabla T$, in turn, leads to a surface tension gradient, $\nabla \sigma$, which is the driving force for an influx of liquid from the meniscus towards the film, i.e., for a stabilizing Marangoni flow.

(iii) The stabilizing force $\nabla \sigma$ acts against three destabilizing factors: the evaporative mass flux, the capillary pressure and the attractive disjoining pressure, all of which take away liquid from the film. The thermal Marangoni effect slows down the thinning of the film and may eventually lead to the establishment of a stationary film thickness similar to the one caused by the Gibbs-Marangoni effect in Marrucci’s theory of coalescence (Marrucci, 1969).

A

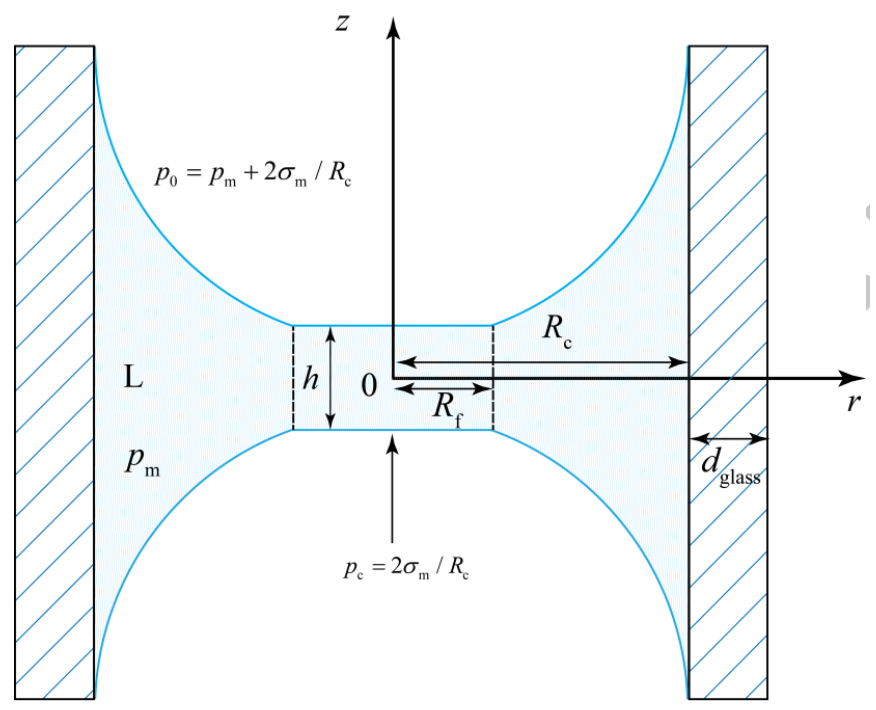

B

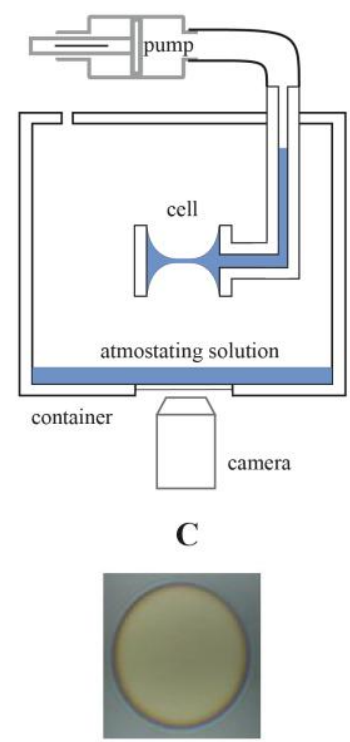

Figure 1. Scheme of a foam film formed in a Scheludko cell. A. A disc-shaped film is in contact with a biconcave meniscus that sticks to the wall of a capillary of inner radius $R_{\mathrm{c}} \sim 2 \mathrm{~mm}$. The pressure $p_{\mathrm{m}}$ in the meniscus is lower than the atmospheric $p_{0}$ by the capillary pressure $2 \sigma_{\mathrm{m}} / R_{\mathrm{c}}$, eq. (2), which causes drainage of the film. B. The capillary is fixed via a glass tube at height of $\sim 1 \mathrm{~cm}$ inside a container, the bottom of which is covered by a layer of 'atmostating liquid' maintaining constant humidity. The container was normally sealed, but in some cases it was opened to expose the film to the humidity of the laboratory. $\mathbf{C}$. Image of a typical aqueous film and its periphery (focussed on the film zone). The colour corresponds to homogeneous thickness of about $h=110 \mathrm{~nm}$; the film radius is $R_{\mathrm{f}}=200 \mu \mathrm{m}$.

A water droplet in dry air is, from Fuchs's eq. 6.6 (Fuchs, 1959), cooler than its environment by $\sim 20 \mathrm{~K}$ once a steady state is reached. A similar temperature difference must occur between the fluid in the meniscus and the ambient gas. The difference between the meniscus and the 
centre of the film is much smaller $(\sim 0.1 \mathrm{~K}$, as will be shown below), yet it is large enough to cause a considerable Marangoni flow, since the surfactant-free liquid surface is highly mobile.

\subsection{Hydrodynamics}

A disc-shaped plane-parallel film in contact with a biconcave droplet (Figure 1) drains under the action of the capillary pressure and, for sufficiently thin films, surface forces. As $h<<R_{\mathrm{f}}$, the lubrication approximation is applicable to the flow within the film (Reynolds, 1886). In addition, the capillary flow approximation can be used as $R e \sim 10^{-9}$.

Most practically observed foam films contain at least trace quantities of surfactants, which lead to nearly complete tangential immobilization of the liquid surface $\left(\left.v_{r}\right|_{z=h / 2}=0\right)$ and film drainage according to the Reynolds law (Radoëv et al., 1974; Sheludko, 1967). In contrast, the films we study consist of a single-component liquid and have free mobile surfaces. Therefore, both the normal and tangential velocities can be non-zero at the surfaces of the film,

$$
\left.v_{r}\right|_{z= \pm h / 2}=V_{r}(r) \quad \text { and }\left.\quad v_{z}\right|_{z= \pm h / 2}=V_{z},
$$

where $V_{\mathrm{z}}$ is independent of $r$. The pressure $p_{\mathrm{m}}$ in the meniscus is lower than $p_{0}$ in the gas phase:

$$
p_{\mathrm{m}}=p_{0}-2 \sigma_{\mathrm{m}} / R_{\mathrm{c}},
$$

where $\sigma_{\mathrm{m}}$ is the tension of the surface of the meniscus. Since $R_{\mathrm{f}}<<R_{\mathrm{c}}$, the meniscus (which has the shape of a nodoid) is almost spherical and the capillary pressure is $p_{\mathrm{c}}=2 \sigma_{\mathrm{m}} / R_{\mathrm{c}}$ to a good approximation (Sheludko, 1967). The capillary pressure drives the drainage of the film, which is counteracted by the Marangoni effect dictated by the evaporation-induced surface tension gradient $\mathrm{d} \sigma \mathrm{d} r$. The latter exerts a tangential force at the film surface, which is balanced by the rz-component of the viscous stress tensor (Levich, 1962; Radoëv et al., 1974):

$$
\frac{\mathrm{d} \sigma}{\mathrm{d} r}=\left.\eta \frac{\partial v_{r}}{\partial z}\right|_{z=h / 2},
$$

where $\eta$ is the viscosity of the liquid. The gradient $\mathrm{d} \sigma \mathrm{d} r$ originates from the difference between the heat capacities of the meniscus and the film, which results into different cooling rates of the two regions and, therefore, a temperature gradient $\nabla T^{\mathrm{L}}$ in the film. For a liquid far from its critical temperature, the dependence of $\sigma$ on $T^{\mathrm{L}}$ is linear (Palit, 1956):

$$
\sigma(r)=\sigma_{\mathrm{m}}-s^{\mathrm{s}}\left(T^{\mathrm{L}}(r)-T_{\mathrm{m}}\right)
$$


where $T^{\mathrm{L}}(r)$ is the temperature profile in the film, $s^{\mathrm{S}}=-\partial \sigma \partial T$ is the surface entropy of the liquid, and $T_{\mathrm{m}}$ is the temperature of the meniscus.

Using the above relations as boundary conditions for the Navier-Stokes equations, the tangential and normal velocities $v_{r}$ and $v_{z}$, the pressure distribution $p(r)$ and the radial surface velocity $V_{r}$ of the film can be expressed with $T^{\mathrm{L}}(r)$ and $V_{z}$ :

$$
\begin{aligned}
& v_{r}=-\frac{h^{2}-4 z^{2}}{8 \eta} \frac{\mathrm{d} p}{\mathrm{~d} r}+V_{r}(r), \\
& v_{z}=\frac{3 h^{2} z-4 z^{3}}{24 \eta} \frac{1}{r} \frac{\mathrm{d}}{\mathrm{d} r}\left(r \frac{\mathrm{d} p}{\mathrm{~d} r}\right)-\frac{z}{r} \frac{\mathrm{d} r V_{r}(r)}{\mathrm{d} r}, \\
& p(r)=p_{\mathrm{m}}-\frac{2}{h} s^{\mathrm{S}}\left(T^{\mathrm{L}}(r)-T_{\mathrm{m}}\right), \\
& V_{r}(r)=-\frac{h s^{\mathrm{S}}}{6 \eta} \frac{\partial T^{\mathrm{L}}}{\partial r}-\frac{r}{h} V_{z} .
\end{aligned}
$$

these expressions are derived in S2; the derivation is similar to that for other film drainage problems, see e.g. (Radoëv et al., 1974).

\subsection{Heat and mass transfer}

The evaporation of the biconcave droplet causes cooling of the liquid in the film and the meniscus. However, the meniscus remains warmer due to the thermostating effect of both the large quantity of liquid there and the glass holder. The resulting temperature gradient is controlled by the balance between the heat lost due to evaporation and the heat flux $Q$ through the gas phase (we show in S3.5 that the heat flux through the liquid is negligible).

We assume that the temperature $T_{\mathrm{m}}$ of the meniscus is homogeneous, whereas the temperature of the film is a quadratic function of $r$. Thus, the difference between the temperature of the surface and the ambient air, $\Delta T(r)=T^{\mathrm{L}}-T_{\infty}$, obeys

$$
\Delta T=\left\{\begin{array}{cc}
\Delta T_{\mathrm{m}}+\frac{T_{r r}}{2}\left(r^{2}-R_{\mathrm{f}}^{2}\right), & \text { film surface } \\
\Delta T_{\mathrm{m}}, & \text { meniscus surface }
\end{array}\right.
$$

Here, $\Delta T_{\mathrm{m}}=T_{\mathrm{m}}-T_{\infty}$ is the difference in temperature between the meniscus and the surrounding gas, and $T_{r r}$ is a coefficient which we will determine later on. The assumption for parabolic $T(r)$ is analogous to the parabolic approximation for the profile of the temperature inside an 
evaporating spherical droplet (e.g., (Dombrovsky and Sazhin, 2003; Snegirev, 2013)) and is implicitly or explicitly used for many problems for thin film drainage problems that involve Gibbs- and electro-Marangoni effects - e.g., the integration with respect to $r$ of eqs. 25a-b in (Valkovska and Danov, 2001) leads to parabolic profiles of the adsorption and the electrical potential in the film. It can be expected that a better approximation of the $T$ profile $\left(\mathrm{a} 4^{\text {th }}\right.$-order polynomial) will lead to corrections of $\mathrm{O}\left(h^{2} / R_{\mathrm{f}}^{2}\right)$ in the results for the fluxes that follow.

We assume that the vapour concentration at the surface of the liquid follows the temperature profile (9) via the Clausius-Clapeyron equation,

$$
C^{\mathrm{S}}=\frac{p^{\mathrm{o}}}{R T^{\mathrm{L}}} \exp \left[-\frac{h_{\mathrm{e}}}{R}\left(\frac{1}{T^{\mathrm{L}}}-\frac{1}{T_{\mathrm{e}}^{\mathrm{o}}}\right)\right]
$$

where $p^{\circ}=101325 \mathrm{~Pa}$ is the standard pressure; $T_{\mathrm{e}}^{\circ}$ is the temperature of boiling of the liquid at $p^{\circ} ; h_{\mathrm{e}}$ is its heat of evaporation, and $R$ is the gas constant. We now substitute $T^{\mathrm{L}}$ with $T_{\mathrm{m}}+\delta T(r)$, where $\delta T=1 / 2 T_{r r}\left(r^{2}-R_{\mathrm{f}}^{2}\right)$ is the temperature difference between the film and the meniscus, see eq. (9). Since $\delta T \ll T_{\mathrm{m}}$, we can expand eq. (10) in a series, which yields:

$$
\Delta C^{\mathrm{S}}=\left\{\begin{array}{cc}
\Delta C_{\mathrm{m}}+\frac{C_{r r}}{2}\left(r^{2}-R_{\mathrm{f}}^{2}\right)+\mathrm{O}\left(\delta T^{2}\right), & \text { film surface; } \\
\Delta C_{\mathrm{m}}, & \text { meniscus surface }
\end{array}\right.
$$

Here, we used the symbols

$$
\begin{aligned}
\Delta C^{\mathrm{S}} & =C^{\mathrm{S}}-C_{\infty} ; \quad \Delta C_{\mathrm{m}}=C_{\mathrm{eq}}-C_{\infty} ; \\
C_{\mathrm{eq}} & =\frac{p^{\mathrm{o}}}{R T_{\mathrm{m}}} \exp \left[-\frac{h_{\mathrm{e}}}{R}\left(\frac{1}{T_{\mathrm{m}}}-\frac{1}{T_{\mathrm{e}}^{\mathrm{o}}}\right)\right] ; \\
C_{r r} & =\left(\frac{h_{\mathrm{e}}}{R T_{\mathrm{m}}}-1\right) C_{\mathrm{eq}} \frac{T_{r r}}{T_{\mathrm{m}}},
\end{aligned}
$$

where $C_{\mathrm{eq}}$ is the Clausius-Clapeyron vapour concentration for the temperature of the meniscus, and $C_{r r}$ is a quantity analogous to $T_{r r}$ : the coefficient of the second-degree term of the concentration profile $C^{\mathrm{S}}(r)$ at the surface of the film. $C_{\infty}$ is the ambient vapour concentration far from the biconcave droplet, which is related to the relative saturation $x$ as:

$$
x=C_{\infty} / C_{\mathrm{eq}}\left(T_{\mathrm{m}}=T_{\infty}\right)=C_{\infty} / C_{\mathrm{eq} 0} .
$$

Note that $x$ is defined with respect to $C_{\mathrm{eq} 0}$, the equilibrium vapour concentration at the ambient temperature. 
The evaporating meniscus acts as a vapour source and a heat sink perturbing the concentration and temperature profile from the homogeneous distribution, i.e., it leads to the formation of diffusion and thermal boundary layers. The characteristic length of these layers is of the order of the size of the biconcave droplet, $R_{\mathrm{c}} \sim 2 \mathrm{~mm}$, as in the problem for an evaporating spherical drop (Fuchs, 1959). This complicates the model because one must consider the concentration and temperature profiles outside the glass capillary and explains why Manev (Manev, 2013) found that the position of the holder within the container influences the process of evaporation. An exact model would require a detailed consideration of the geometry of the holder and the container; such a model would yield a result specific to the construction of the cell and to the shape of the meniscus and will be of little value in terms of generality. Instead of adopting such a treatment, we will use dimensional analysis to obtain a general dependence of the mass flux from the film to the environment $(J)$ and the heat flux from the air towards the film $(Q)$ on all relevant parameters. In $\mathrm{S} 3 \& 4$, we formulate a crude model of the geometry of the system, which allows the missing numerical factors in this dependence to be estimated. Naturally, this approach restricts the validity of the final results for the fluxes to only a correct order of magnitude.

Let us first apply dimensional analysis in order to derive the total diffusion flux $J$ through the film surfaces. According to eq. (11), the vapour concentration near the film region is perturbed by two additive effects: $\Delta C_{\mathrm{m}}$ due to the cooling of the biconcave droplet as a whole and $1 / 2 C_{r r}\left(r^{2}-R_{\mathrm{f}}^{2}\right)$, due to the additional cooling of the film region. The concentration gradient due to the cooling of the biconcave droplet drives a diffusive flux of density $j_{\mathrm{c}}$ proportional to the concentration difference between the meniscus and the surrounding air $\left(\Delta C_{\mathrm{m}}\right.$, see eq. (12)) divided by the characteristic length (the size of the droplet, $\sim R_{\mathrm{c}}$ ) of the respective diffusion layer:

$$
j_{\mathrm{c}} \sim D \Delta C_{\mathrm{m}} / R_{\mathrm{c}}
$$

where $D$ is the diffusion coefficient of the vapours. As for the second perturbation in the film region, it generates a flux $j_{\mathrm{f}}$ proportional to the concentration difference between the vapours above the colder film and those above the warmer meniscus divided by the characteristic length of the perturbation, the film radius $R_{\mathrm{f}}$, i.e., $j_{\mathrm{f}} \sim D\left(\left.C^{\mathrm{S}}\right|_{r=0}-\left.C^{\mathrm{S}}\right|_{r=R \mathrm{f}}\right) / R_{\mathrm{f}}$, From eq. (11) it follows that $\left.C^{\mathrm{S}}\right|_{r=0}-\left.C^{\mathrm{S}}\right|_{r=R \mathrm{f}} \sim-C_{r r} R_{\mathrm{f}}^{2}$, which leads to

$$
j_{\mathrm{f}} \sim-D R_{\mathrm{f}} C_{r r} .
$$


Combining eqs. (17) and (16), we arrive at an expression for the total mass outflux through the surface of the film:

$$
J \sim R_{\mathrm{f}}^{2}\left(j_{\mathrm{f}}+j_{\mathrm{c}}\right)=D R_{\mathrm{f}}^{2}\left(-K_{1} R_{\mathrm{f}} C_{r r}+K_{2} \frac{\Delta C_{\mathrm{m}}}{R_{\mathrm{c}}}\right) .
$$

Here, $K_{1}$ and $K_{2}$ are dimensionless coefficients of value specific to the precise geometry of the system, estimated in $\mathrm{S} 3 \& 4$ based on an approximate model. The final result for $J$ reads

$$
J=D R_{\mathrm{f}}^{2}\left[-\frac{8}{3} R_{\mathrm{f}} C_{r r}+(2-\sqrt{2}) \frac{\pi}{R_{\mathrm{c}}} \Delta C_{\mathrm{m}}\right] ;
$$

$J$ is defined as the total positive diffusive flux through both surfaces of the film. Note that our solution to the considered problem neglects the presence of a transition layer in the concentration profile in the vicinity of the interface (known as a Fuchs layer), as well as convective diffusion we analyse the limitations of these approximations in S5 and 6, respectively; in particular, we show that convective diffusion is significant for highly volatile liquids, e.g. hexane.

The respective heat flux $Q$ from the air through the surfaces of the film, by analogy with eq. (19), is

$$
Q=\kappa R_{\mathrm{f}}^{2}\left[\frac{8}{3} R_{\mathrm{f}} T_{r r}-(2-\sqrt{2}) \frac{\pi}{R_{\mathrm{c}}} \Delta T_{\mathrm{m}}\right],
$$

where $\kappa$ is the thermal conductivity of the air. As with the mass transfer problem, we neglect the transition (Knudsen) layer and the convective terms in the heat transfer equation, cf. S5 and 6. The heat transfer via radiation is also neglected - by analogy with the case of an evaporating droplet (Fuchs, 1959), this assumption should hold true for the film radii that we consider in this paper $(\sim 100 \mu \mathrm{m})$. We have also neglected the term $\partial T / \partial t$ in the heat equation; in $\mathrm{S} 3$, we show that this is justified for films of $h<1 \mu \mathrm{m}$.

\subsection{Heat balance}

The approximate nature of our solution to the heat and mass transfer problems does not permit a local form of the heat balance to be used for our problem. Consequently, in order to determine the unknown coefficient $T_{r r}$ in the temperature profile (9), we will employ an integral heat balance for the film. This approach is widely used in the literature for the force balance in thin films, e.g., the Reynolds law for thin film drainage is obtained through an integral mechanical 
balance that substitutes the local Young-Laplace equation as the latter cannot be fulfilled if the film is assumed to be plane-parallel (Ivanov and Dimitrov, 1974). The integral heat balance states that the total heat consumption rate due to evaporation, $h_{\mathrm{e}} J$, is compensated by the integral heat influx $Q$ through the air:

$$
h_{\mathrm{e}} J=Q,
$$

Substituting here the relations (19) and (20), together with eq. (14) for $C_{r r}$, we obtain an equation for $T_{r r}$. Solving it gives the result

$$
T_{r r}=0.69 \frac{T_{\mathrm{m}}}{R_{\mathrm{f}} R_{\mathrm{c}} C_{\mathrm{eq}}} \frac{\Delta C_{\mathrm{m}}+\frac{\kappa \Delta T_{\mathrm{m}}}{h_{\mathrm{e}} D}}{\frac{h_{\mathrm{e}}}{R T_{\mathrm{m}}}-1+\frac{\kappa T_{\mathrm{m}}}{h_{\mathrm{e}} D C_{\mathrm{eq}}}} .
$$

The term proportional to $\Delta T_{\mathrm{m}}$ in the numerator can in principle nullify $T_{r r}$ - that is, there exists a temperature difference between the meniscus and the ambient air, $\Delta T_{\mathrm{m}}=-h_{\mathrm{e}} D \Delta C_{\mathrm{m}} / \kappa$, such that the temperature drop in the film and the associated Marangoni effect vanish. Thus, once the meniscus becomes cold enough, the thin films can no longer be stabilized by the evaporation.

\subsection{The cooling of the meniscus}

The time dependence of the temperature of the meniscus $T_{\mathrm{m}}$ follows from the heat balance for the glass capillary and the liquid in the meniscus. This balance involves the heat flux $Q_{\mathrm{m}}$ coming from the air through both surfaces of the meniscus and the respective evaporative flux $J_{\mathrm{m}}$. Applying dimensional analysis as done for $J$ above, one can show that $Q_{\mathrm{m}} \propto-\kappa R_{\mathrm{c}} \Delta T_{\mathrm{m}}$. The numerical coefficient in this relation is estimated in S5:

$$
Q_{\mathrm{m}}=-4.57 \kappa R_{\mathrm{c}} \Delta T_{\mathrm{m}} .
$$

The mass transfer problem leads to a similar expression for the diffusive flux $J_{\mathrm{m}}$ through the surfaces of the meniscus:

$$
J_{\mathrm{m}}=4.57 D R_{\mathrm{c}} \Delta C_{\mathrm{m}} .
$$

We make the additional assumption that at all times, the meniscus and the glass of the capillary are in thermal equilibrium. In this approximation, the rate of change of $T_{\mathrm{m}}$ is determined by the rate of heat loss due to evaporation $\left(h_{\mathrm{e}} J_{\mathrm{m}}\right)$, and the rate at which heat is gained from the gas phase $\left(Q_{\mathrm{m}}\right)$ : 


$$
C_{p} \frac{\mathrm{d} T_{\mathrm{m}}}{\mathrm{d} t}=Q_{\mathrm{m}}-h_{\mathrm{e}} J_{\mathrm{m}}=-4.57 R_{\mathrm{c}}\left(\kappa \Delta T_{\mathrm{m}}+h_{\mathrm{e}} D \Delta C_{\mathrm{m}}\right)
$$

where $C_{p}$ is the sum of the heat capacities of the liquid and the part of the glass holder in direct contact with the fluid. Its value is estimated in S8 - for water films in a borosilicate capillary of diameter $R_{\mathrm{c}}=2 \mathrm{~mm}$ and wall thickness $d_{\text {glass }}=1 \mathrm{~mm}, C_{p} \approx 0.18 \mathrm{~J} / \mathrm{K}$.

The right-hand side of eq. (25) can become zero, which means that a stationary temperature difference between the meniscus and the air $\left(\Delta T_{\mathrm{m}, \mathrm{st}}\right)$ will be reached eventually, as in the case of a spherical droplet (Fuchs, 1959). This stationary temperature decreases with the decrease of the ambient relative saturation $x$. To analyse the dependence of $\Delta T_{\mathrm{m}, \mathrm{st}}$ on $x$, we set the right-hand side of eq. (25) to zero and rearrange it using eqs. (12)-(13):

$$
C_{\infty}=x C_{\mathrm{eq} 0}=C_{\mathrm{eq}}+\frac{\kappa \Delta T_{\mathrm{m}, \mathrm{st}}}{h_{\mathrm{e}} D}=\frac{p^{0}}{R\left(\Delta T_{\mathrm{m}, \mathrm{st}}+T_{\infty}\right)} \exp \left[-\frac{h_{\mathrm{e}}}{R}\left(\frac{1}{\Delta T_{\mathrm{m}, \mathrm{st}}+T_{\infty}}-\frac{1}{T_{\mathrm{e}}^{\mathrm{o}}}\right)\right]+\frac{\kappa \Delta T_{\mathrm{m}, \mathrm{st}}}{h_{\mathrm{e}} D},
$$

where we used that, by definition, $x=C_{\infty} / C_{\mathrm{eq} 0}$. Instead of the dependence $\Delta T_{\mathrm{m}, \mathrm{st}}(x)$, this result determines the inverse function, $x\left(\Delta T_{\mathrm{m}, \mathrm{st}}\right)$; it is plotted in Fig $\mathrm{S}$ 9. According to eq. (26), for the experimentally relevant undersaturations, a water meniscus is colder than the ambient air by 5-10 $\mathrm{K}$ at the stationary state. The stationary temperature difference predicted by the equation above is precisely the value at which the numerator in eq. (22) for $T_{r r}$ becomes zero - that is, when the temperature drop in the meniscus reaches $\Delta T_{\mathrm{m}, \mathrm{st}}$, the Marangoni effect disappears. However, as we will show next, the films become unstable even before $T_{r r}$ reaches zero.

The dependence of $\Delta T_{\mathrm{m}}$ on time at a given saturation $x$ follows from the heat balance (25):

$$
t=-\frac{C_{p}}{4.57 R_{\mathrm{c}}} \int_{0}^{\Delta T_{\mathrm{m}}} \frac{\mathrm{d} \Delta T_{\mathrm{m}}}{\kappa \Delta T_{\mathrm{m}}+h_{\mathrm{e}} D \Delta C_{\mathrm{m}}}
$$

where as an initial condition we have used that at $t=0$, the temperature of the meniscus is equal to that of the surrounding air. The integral (27) is not analytical due to the complicated dependence of $\Delta C_{\mathrm{m}}$ on $\Delta T_{\mathrm{m}}$, see eqs. (12)-(13). In $\mathrm{S} 8$, we use a series expansion to obtain an analytical expression valid for small $\Delta T_{\mathrm{m}}$ and for $x$ close to 1 .

\subsection{Drainage and stability of an evaporating film.}

Having derived the expression (22) for $T_{r r}$, we can now explicitly determine the drainage velocity of the film. In order to do so, we will formulate an integral balance of the forces acting 
in the normal direction, by analogy with the derivation of the Reynolds law for drainage of films of tangentially immobile surfaces (Reynolds, 1886). The integral approach is necessary because our model states in advance that the studied film is plane-parallel (based on the experiment, Figure 1C), thereby making the local form of the normal force balance inapplicable (Ivanov and Dimitrov, 1974). Employing a local boundary condition would be tantamount to solving the Young-Laplace equation for the film shape (Manev et al., 1997; Tsekov and Ruckenstein, 1994), and goes beyond the aims of our study. The integral normal force balance states that the total force acting on the film in normal direction is zero,

$$
\left.2 \pi \int_{0}^{R_{\mathrm{f}}}\left(p_{0}-p(r)-\Pi+2 \eta \frac{\partial v_{z}}{\partial z}\right)\right|_{z=h / 2} r \mathrm{~d} r=0 .
$$

The balance includes the pressure $p_{0}$ in the gas, the hydrodynamic pressure $p(r)$, the viscous force in the liquid, $-2 \eta \partial v_{z} / \partial z$, and the disjoining pressure $\Pi$ (the surface forces) in the film. Vapour recoil force can be neglected for the low to moderate evaporation rates we consider (Oron et al., 1997).

The precise nature of the surface forces acting in films formed from pure liquids is unclear. We assume that the main contribution to $\Pi$ is the van der Waals $h^{-3}$ attraction,

$$
\Pi=\Pi_{\mathrm{vdW}}=-A_{\mathrm{H}} / 6 \pi h^{3},
$$

while the surface charge of the pure liquid and the respective electrostatic disjoining pressure $\Pi_{\mathrm{el}}$ (Sheludko, 1967) are neglected. However, there is evidence that $\Pi_{\mathrm{el}}$ is a significant factor for the stability of pure water films (Exerowa and Kruglyakov, 1998; Yaminsky et al., 2010b), and the surface of pure water is, in fact, charged (Kolarov et al., 1993; Stubenrauch and Klitzing, 2003). Manica and Chan (Manica and Chan, 2011) modelled the disjoining pressure in films formed from distilled water as having a repulsive electrostatic component only. They obtained good agreement with experimental data for $h(t)$, but to achieve that they had to assume that the air|water surface is tangentially immobile. We have two reasons for leaving the question of $\Pi_{\mathrm{el}}$ aside. First, the experimentally observed evaporating saline films in (Karakashev et al., 2008) exhibit considerable stability at electrolyte concentrations of over $1 \mathrm{M}$, where electrostatic forces are completely screened - this means that even if $\Pi_{\mathrm{el}}$ contributes to the stabilization, there must be another, dynamic stabilizing factor. Our second reason is that, for pure water films, the precise dependence of $\Pi_{\mathrm{el}}$ on the film thickness $h$ is unknown. In particular, it is unlikely that $\Pi_{\mathrm{el}}$ follows 
the DLVO theory, the least reason being that the ionic strength of pure water $\left(10^{-7} \mathrm{M}\right)$ corresponds to an average distance between two ions that is greater than the experimentally observed $h$, which violates one of the assumptions under which the DLVO formula is derived, see Derjaguin (Derjaguin, 1986): for the mean-field potential that appears in the PoissonBoltzmann distribution. In addition, as the Debye length is greater than $h$, the repulsion can be expected to act in the charge regulation regime, which requires an adsorption isotherm for these ions. The choice of an isotherm is complicated by the fact that there is no satisfactory explanation of the origin of the surface charge that produces the repulsion - for example, it seems rather far-fetched that hydroxide ions could adsorb at the air|water interface with an adsorption energy of the order of $20 \times k_{\mathrm{B}} T$ (reported in (Marinova et al., 1996)), i.e., comparable to that of sodium dodecyl sulfate (Slavchov et al., 2014). Other effects, such as the image force contribution to the disjoining pressure, become important at such low concentrations (Boinovich, 2010; Torrie et al., 1984)[. Nevertheless, we performed the whole analysis with the inclusion of the DLVO expression for $\Pi_{\mathrm{el}}$ in $\mathrm{S} 7$, in the constant potential and the constant charge regimes, as well as in the asymptotic form employed by Yaminsky (Yaminsky et al., 2010b). However, in view of the arguments stated above, we are sceptical towards its reliability and in the following sections we only consider the van der Waals term (29) in the disjoining pressure. In the worst case, the neglect of $\Pi_{\mathrm{el}}$ makes our model applicable for non-aqueous films only, as there is little evidence of surface charge at the oil surface. It should be pointed out however, that eq. (29) itself is approximate for it neglects the retardation effect - the latter is known to be important for oil films (Israelachvili, 2011; Scheludko et al., 1965).

Substituting the expressions (7),(2),(9),(6),(8) and (29) for $p(r), p_{\mathrm{m}}, T(r), v_{z}, V_{r}$ and $\Pi$ in the force balance (28), we obtain an explicit equation for $V_{z}$. Its solution reads:

$$
V_{z}=\frac{R_{\mathrm{f}}^{2} s^{\mathrm{S}} T_{r r}}{8 \eta}\left(1-\frac{4}{3} \frac{h^{2}}{R_{\mathrm{f}}^{2}}\right)-\frac{h}{4 \eta}\left(p_{\mathrm{c}}-\Pi_{\mathrm{vdW}}\right) \approx \frac{R_{\mathrm{f}}^{2} s^{\mathrm{s}} T_{r r}}{8 \eta}-\frac{h}{\eta} \frac{\sigma_{\mathrm{m}}}{2 R_{\mathrm{c}}}-\frac{1}{\eta} \frac{A_{\mathrm{H}}}{24 \pi h^{2}},
$$

where we used that $h / R_{\mathrm{f}} \ll 1$. With knowledge of the drainage velocity $V_{z}$, we can proceed with the analysis of the film drainage velocity $\mathrm{d} h / \mathrm{d} t$, which follows from the mass balance of the liquid in the film:

$$
\frac{\mathrm{d} h}{\mathrm{~d} t}=-\frac{v^{\mathrm{L} J}}{\pi R_{\mathrm{f}}^{2}}+2 V_{z} \approx \frac{R_{\mathrm{f}}^{2} s^{\mathrm{S}} T_{r r}}{4 \eta}-\frac{h}{\eta} \frac{\sigma_{\mathrm{m}}}{R_{\mathrm{c}}}-\frac{A_{\mathrm{H}}}{12 \pi \eta h^{2}},
$$


where $v^{\mathrm{L}}$ is the molar volume of the liquid. The first term in eq. (31) reflects the loss of fluid caused by evaporation and the term $2 V_{z}$ quantifies the normal volume flux density towards the two surfaces of the film. According to eq. (30), there are three factors that influence $V_{z}-$ the first term $\left(\propto T_{r r}\right)$ is due to the Marangoni effect, which causes liquid to flow from the meniscus to the film, while the second $\left(\propto \sigma_{\mathrm{m}} / R_{\mathrm{c}}\right)$ and the third $\left(\propto A_{\mathrm{H}}\right)$ describe the flow of liquid outward of the film due to the capillary pressure and the disjoining pressure, respectively. For water films of $R_{\mathrm{f}}$ $=150 \mu \mathrm{m}$ in contact with a thermostated meniscus with the parameters specified in $\mathrm{S} 1$, we obtain the following estimates: $v^{\mathrm{L}} J / \pi R_{\mathrm{f}}^{2} \sim 10^{-8} \mathrm{~m} / \mathrm{s}$ for the diffusive term (using eq. (19)), $R_{\mathrm{f}}^{2} s^{\mathrm{S}} T_{r r} / 4 \eta \sim$ $10^{-2} \mathrm{~m} / \mathrm{s}$ for the term due to the Marangoni flux, $h \sigma_{\mathrm{m}} / \eta R_{\mathrm{c}} \sim 10^{-2} \mathrm{~m} / \mathrm{s}$ for the capillary pressure term and $A_{\mathrm{H}} / 12 \pi \eta h^{2} \sim 10^{-5} \mathrm{~m} / \mathrm{s}$ for the one caused by the van der Waals attraction. Thus, somewhat contrary to what one might intuitively expect, the mass loss due to evaporation has a minor impact on the rate of thinning $\mathrm{d} h / \mathrm{d} t$. A similar result has been obtained for an evaporating sessile droplet (Tsoumpas et al., 2015). The evolution of the film is thus predominantly controlled by the Marangoni flux, the disjoining and the capillary pressure.

One can use eq. (31) to derive the dependence of $h$ on $t$; the result is discussed in S6. There, the characteristic time for reaching the stationary thickness of the film is predicted to be of the order of $10^{-6} \mathrm{~s}$. Here, we analyse only the stationary state itself. Setting the right-hand side of eq. (31) to zero and rearranging, we arrive at the following equation for the stationary thickness:

$$
\frac{h}{h_{\mathrm{cr}}}+\frac{h_{\mathrm{cr}}^{2}}{2 h^{2}}=\frac{k}{2}
$$

where the symbols $k$ and $h_{\mathrm{cr}}$ stand for

$$
\begin{aligned}
& k=\frac{1}{2} \frac{R_{\mathrm{f}}^{2}}{h_{\mathrm{cr}}} \frac{R_{\mathrm{c}} s^{\mathrm{s}} T_{r r}}{\sigma_{\mathrm{m}}}=0.92 \frac{R_{\mathrm{f}}}{R_{\mathrm{c}}^{1 / 3}} \frac{s^{\mathrm{s}} T_{\mathrm{m}}}{A_{\mathrm{H}}^{1 / 3} \sigma_{\mathrm{m}}^{2 / 3}} \frac{1-x \frac{C_{\mathrm{eq} 0}}{C_{\mathrm{eq}}}+\frac{\kappa \Delta T_{\mathrm{m}}}{h_{\mathrm{e}} D C_{\mathrm{eq}}}}{\frac{h_{\mathrm{e}}}{R T_{\mathrm{m}}}-1+\frac{\kappa T_{\mathrm{m}}}{h_{\mathrm{e}} D C_{\mathrm{eq}}}} \text { and } \\
& h_{\mathrm{cr}}=\left(\frac{A_{\mathrm{H}} R_{\mathrm{c}}}{6 \pi \sigma_{\mathrm{m}}}\right)^{1 / 3} .
\end{aligned}
$$

There are two physically meaningful solutions to eq. (32) for the stationary thickness,

$$
h_{\mathrm{st} 1}=\frac{h_{\mathrm{cr}}}{6}\left(a^{1 / 3}+\frac{k^{2}}{a^{1 / 3}}+k\right) \text { and } h_{\mathrm{st} 2}=-\frac{h_{\mathrm{cr}}}{12 a^{1 / 3}}\left[\left(a^{1 / 3}-k\right)^{2}+\mathrm{i} \sqrt{3}\left(a^{2 / 3}-k^{2}\right)\right] \text {, }
$$


where $\mathrm{i}$ is the imaginary unit and the coefficient $a$ is defined as

$$
a=-54+k^{3}+6 \sqrt{81-3 k^{3}} .
$$

Note that $a$ can be complex and $h_{\mathrm{st} 2}$ has real values for a certain range of $k$. Note also that the solutions (35) are quasistationary, as they depend on the coefficient $T_{r r}$, which depends on the temperature of the meniscus (see eq. (22)), and it is therefore a function of time.

\subsection{The limiting case of a thermostated meniscus.}

Let us first consider the limiting case in which the temperature of the meniscus is equal to the ambient one $\left(\Delta T_{\mathrm{m}}=0\right)$. Under this assumption, the thicknesses $h_{\mathrm{st1}}$ and $h_{\mathrm{st2}}(35)$ correspond to a true stationary state, rather than a quasistationary one. The solutions (35) are plotted in Figure 2 - this is the stability diagram of pure water films in contact with thermostated meniscus. The first stationary solution $h_{\text {st } 1}$ gives the stable branch of the diagram, i.e., for any initial thickness $h_{0}$ of the film for which $h_{0}>h_{\mathrm{st} 2}$, it is expected that $h(t \rightarrow \infty)=h_{\mathrm{st} 1}$. Once the film reaches the stable thickness $h_{\text {st } 1}$, it remains stationary as long as the meniscus does not cool down. The branch $h_{\mathrm{st} 2}$ of eq. (35) corresponds to an unstable stationary state. A film of initial thickness $h_{0}=h_{\mathrm{st} 2}+\delta$, where $\delta$ is a positive perturbation, will thicken until the stable branch is reached $\left(h=h_{\mathrm{st1}}\right)$, while if $h_{0}$ is smaller than $h_{\mathrm{st} 2}$, the film is expected to drain until rupture (S6, Figure S2). Our eq. (32) and Figure 2 are analogous to Marrucci's eq. 16 and fig. 2 (Marrucci, 1969) that represent a similar balance between the concentration-driven Marangoni effect, the capillary pressure and the van der Waals force.

The mass balance (32) only permits a stationary state if the relative saturation $x$ is below a certain critical value, $x_{\text {cro }}$, i.e., if the evaporation is intense enough. This critical value corresponds to the maximum of $x$ as a function of $h_{\mathrm{st}}$. There, the film thickness is precisely equal to the critical value $h_{\text {cr }}$ from eq. (34), see Figure 2; this must be the smallest observable thickness of the film (for water, it is $h_{\mathrm{cr}}=40.1 \mathrm{~nm}$, see S1 for the parameter values). Substituting $h=h_{\text {cr }}$ in eq. (32), we obtain that the respective critical value of $k$ is 3. Eq. (33) then leads to the critical value of $T_{r r}$ for a thermostated meniscus:

$$
T_{r r 0, \mathrm{cr}}=\frac{6^{2 / 3} A_{\mathrm{H}}^{1 / 3} \sigma_{\mathrm{m}}^{2 / 3}}{\pi^{1 / 3} s^{\mathrm{S}} R_{\mathrm{f}}^{2} R_{\mathrm{c}}^{2 / 3}} .
$$


Equating the right-hand side of eq. (37) with that of (22), using the definitions (12) and (15) and solving for $x$, we obtain the sought value of the critical saturation for a thermostated meniscus:

$$
x_{\mathrm{cr} 0}=1-3.27 \frac{R_{\mathrm{c}}^{1 / 3}}{R_{\mathrm{f}}} \frac{A_{\mathrm{H}}^{1 / 3} \sigma_{\mathrm{m}}^{2 / 3}}{s^{\mathrm{S}} T_{\infty}}\left(\frac{h_{\mathrm{e}}}{R T_{\infty}}-1+\frac{\kappa T_{\infty}}{h_{\mathrm{e}} D C_{\mathrm{eq} 0}}\right) .
$$

At relative saturations $x$ greater than $x_{\mathrm{cr} 0}\left(91 \%\right.$ for pure water films of $\left.R_{\mathrm{f}}=150 \mu \mathrm{m}\right)$, no stationary thickness exists because the evaporation-driven Marangoni flow is too weak to maintain the stationary state. At $x<x_{\mathrm{cr} 0}$, the stable stationary film thickness $h_{\mathrm{st} 1}$ is given by eq. (35). Our critical relative saturation is analogous to the critical concentration occurring in Marrucci’s coalescence model (Marrucci, 1969).

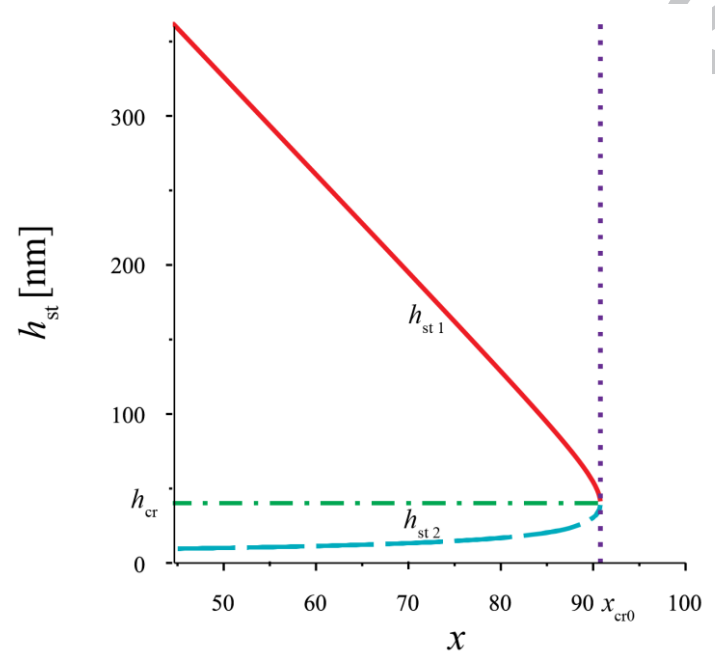

Figure 2. Stationary thickness $h_{\text {st }}$ of a water film as a function of the relative saturation: a stability diagram for a thermostated meniscus, eqs. (35). A stable stationary thickness $h_{\text {st1 }}$ (solid line), exists for humidities lower than the critical value $x_{\mathrm{cr} 0}$. The branch $h_{\mathrm{st} 2}$ (dashed line) is unstable, i.e., a film of initial thickness $h_{0}>h_{\mathrm{st} 2}$ thickens to $h_{\mathrm{st} 1}$, while if $h_{0}<h_{\mathrm{st} 2}$, the film drains until rupture. All films are unstable at $x$ $>x_{\mathrm{cr} 0}$. At $x=x_{\mathrm{cr} 0}$ (dotted vertical line), the quasistationary film thickness is equal to $h_{\mathrm{cr}}$ (dashdot line).

\subsection{Evaporating thin film in contact with a cold meniscus}

The assumption that the meniscus is thermostated leads to the conclusion that evaporating thin water films have an infinite lifetime at $x<x_{\mathrm{cr} 0}$, which contradicts the experimental data we have gathered (see Section 3). This contradiction is resolved when the cooling of the meniscus is accounted for. Let us therefore consider the time dependence of the thickness of a film in contact with a meniscus that cools down following eq. (27). The quasi-stationary film thickness depends on time through $\Delta T_{\mathrm{m}}$. We plot parametrically the stable branch of eq. (35), $h_{\mathrm{st} 1}\left(\Delta T_{\mathrm{m}}\right)$, against 
$t\left(\Delta T_{\mathrm{m}}\right)$ from eq. (27) at several values of the relative saturation; the resulting curves are shown in Figure 3. As the meniscus gradually cools down (Fig. S8), the film remains in a quasistationary state, but its thickness decreases according to eq. (35) in unison with the drop in $T_{\mathrm{m}}$. The thinning process continues until the film reaches the critical thickness (34), whereupon rupture occurs. It is evident from Figure 3 that the expected lifetime of pure water films strongly depends on the relative humidity of the gas in the Scheludko cell: at $x>x_{\mathrm{cr} 0}$, no stationary state is possible; for $x$ $<x_{\mathrm{cr} 0}$, the quasistationary thickness decreases from an initial value of the order of $\sim 100-250 \mathrm{~nm}$ to $h_{\mathrm{cr}}$ over a time period of $\tau \sim 100 \mathrm{~s}$.

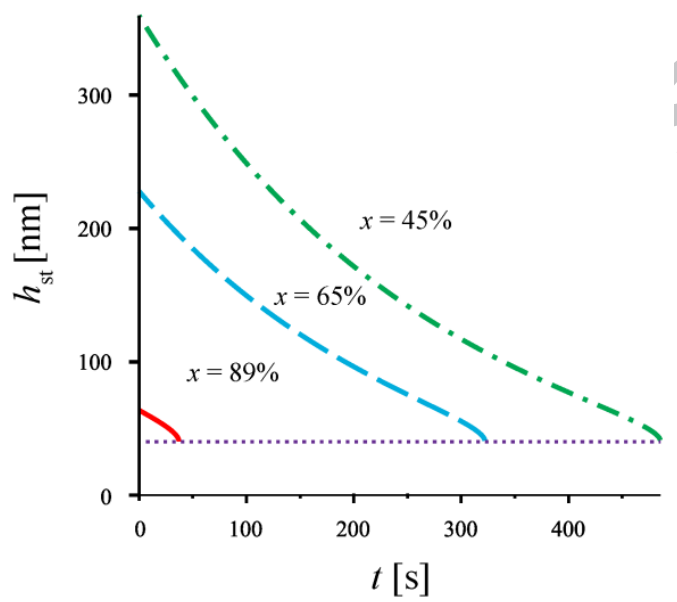

Figure 3. Quastistationary film thickness $h_{\mathrm{st}}$ of an evaporating water film in contact with a nonthermostated meniscus as a function of time $t$ for various values of the relative saturation $x$, at a film radius $R_{\mathrm{f}}=150 \mu \mathrm{m}$. The plot is parametric, using $h_{\mathrm{st} 1}\left(\Delta T_{\mathrm{m}}\right)$ from eq. (35) and $t\left(\Delta T_{\mathrm{m}}\right)$ from eq. (27). The films thin down to the critical thickness $h_{\mathrm{cr}}=40.1 \mathrm{~nm}$ (eq. (34), dotted line), whereupon they are predicted to rupture. See $\mathrm{S} 1$ for the values of the parameters.

We now turn to the dependence of the lifetime $\tau$ of the film on the saturation $x$, which can be obtained via another parametric plot. The critical value of $k$ corresponding to $h_{\mathrm{st} 1}=h_{\mathrm{cr}}$ following from eq. (32) is $k_{\mathrm{cr}}=3$; we substitute this value in eq. (33) and solve it for $x$ :

$$
x_{\mathrm{cr}}=\frac{C_{\mathrm{eq}}}{C_{\mathrm{eq} 0}}+\frac{\kappa \Delta T_{\mathrm{m}}}{h_{\mathrm{e}} D C_{\mathrm{eq} 0}}-3.27 \frac{R_{\mathrm{c}}^{1 / 3}}{R_{\mathrm{f}}} \frac{A_{\mathrm{H}}^{1 / 3} \sigma_{\mathrm{m}}^{2 / 3}}{s^{\mathrm{s}} T_{\mathrm{m}}} \frac{C_{\mathrm{eq}}}{C_{\mathrm{eq} 0}}\left(\frac{h_{\mathrm{e}}}{R T_{\mathrm{m}}}-1+\frac{\kappa T_{\mathrm{m}}}{h_{\mathrm{e}} D C_{\mathrm{eq}}}\right) .
$$

This equation generalizes eq. (38); above the critical humidity $x_{\mathrm{cr}}$, films in contact with a cold meniscus of temperature $T_{\mathrm{m}}$ are predicted to have a finite lifetime $\tau$. To find $\tau$, we substitute eqs. (12)-(13) in eq. (27) for $t\left(\Delta T_{\mathrm{m}}\right)$ (using $C_{\infty}=x C_{\mathrm{eq} 0}$ ); then, we substitute $x$ with its critical value 
(39). The result is $\tau$ as function of $\Delta T_{\mathrm{m}}$. Plotting it parametrically against $x_{\mathrm{cr}}\left(\Delta T_{\mathrm{m}}\right)$ from eq. (39) gives us the sought dependence $\tau(x)$, Figure 4A.

One can infer from eq. (39) that $x_{\mathrm{cr}}$ grows with $R_{\mathrm{f}}$, i.e., the stability of the evaporating film is dependent on its radius. We can rewrite eq. (39) as:

$$
R_{\mathrm{f}}=3.27 \frac{R_{\mathrm{c}}^{1 / 3} A_{\mathrm{H}}^{1 / 3} \sigma_{\mathrm{m}}^{2 / 3}}{s^{\mathrm{s}} T_{\mathrm{m}}} \frac{\frac{h_{\mathrm{e}}}{R T_{\mathrm{m}}}-1+\frac{\kappa T_{\mathrm{m}}}{h_{\mathrm{e}} D C_{\mathrm{eq}}}}{1-x \frac{C_{\mathrm{eq} 0}}{C_{\mathrm{eq}}}+\frac{\kappa \Delta T_{\mathrm{m}}}{h_{\mathrm{e}} D C_{\mathrm{eq}}}} .
$$

According to this formula, films of different radii break at a different value of the temperature $T_{\mathrm{m}}$ of the meniscus, and therefore, have different lifetimes. This is illustrated in Figure 4B, where $R_{\mathrm{f}}\left(\Delta T_{\mathrm{m}}\right)$ is plotted parametrically against $t\left(\Delta T_{\mathrm{m}}\right)$, eqs. (40) and (27), for $x=45 \%$. At this saturation, quasistationary films are expected only for $R_{\mathrm{f}}$ above a critical value $R_{\mathrm{f}, \mathrm{cr}}=$ $R_{\mathrm{f}}\left(\Delta T_{\mathrm{m}}=0\right)$. For a water film formed in a capillary with $R_{\mathrm{c}}=2 \mathrm{~mm}, R_{\mathrm{f}, \text { cr }}$ is equal to $25 \mu \mathrm{m}$, see $\mathrm{S} 1$ for details on the parameters of the system.
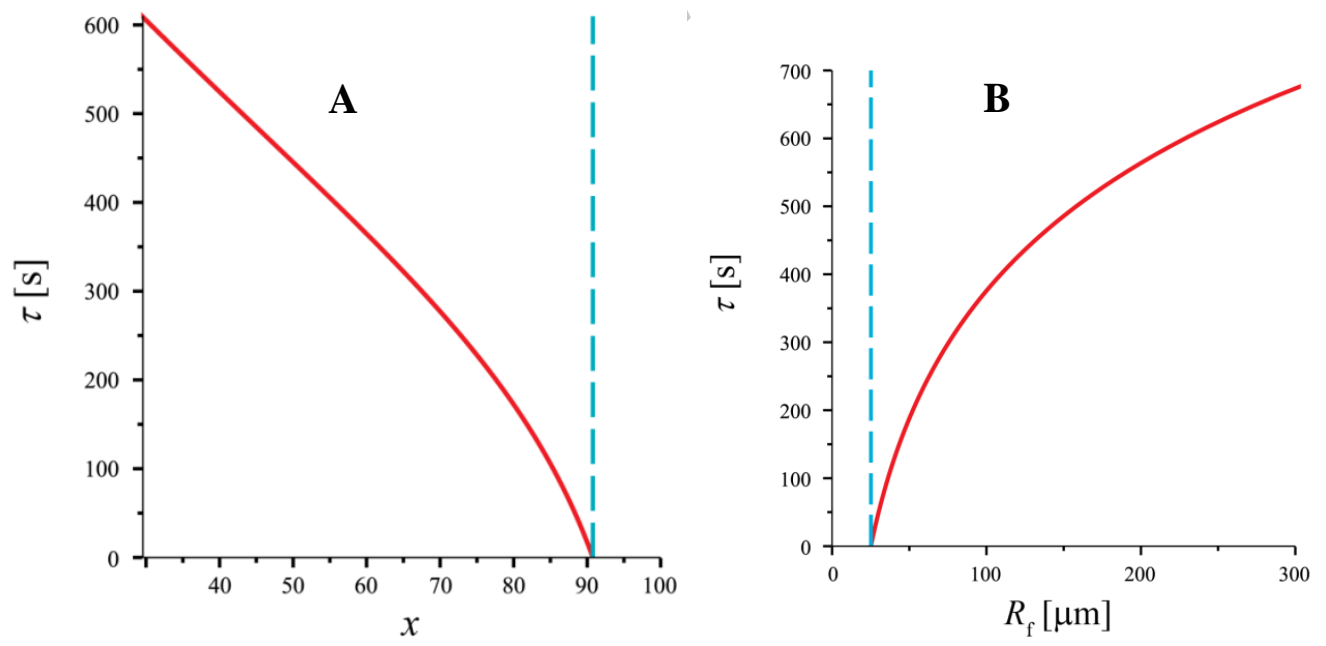

Figure 4. A. Expected lifetime $\tau$ of quastistationary water films of radius $R_{\mathrm{f}}=150 \mu \mathrm{m}$ as a function of the relative saturation $x$ of the ambient gas - parametric plot of $t\left(T_{\mathrm{m}}\right)$ vs. $x_{\mathrm{cr}}\left(T_{\mathrm{m}}\right)$, eqs. (27) and (39). No stationary state is possible at $x>x_{\mathrm{cr} 0}=91 \%$ (eq. (38)). For $x<x_{\mathrm{cr} 0}$ (dashed line), the film lifetime coincides with the time $\tau$ required for the temperature $T_{\mathrm{m}}$ of the meniscus to decrease to the critical value that follows from eq. (39). B. Lifetime of water films as a function of the film radius $R_{\mathrm{f}}$ at $x=45 \%-$ parametric plot of $R_{\mathrm{f}}\left(\Delta T_{\mathrm{m}}\right)$ against $t\left(\Delta T_{\mathrm{m}}\right)$, eqs. (40) and (27). Stationary films are predicted to exist only for $R_{\mathrm{f}}>R_{\mathrm{f}, \mathrm{cr}} \approx 25 \mu \mathrm{m}$. See $\mathrm{S} 1$ for the values of the parameters used. 
The quasistationary approach we use in this section, in which the thickness is a function of time, but $\mathrm{d} h / \mathrm{d} t$ is still equal to 0 , is applicable because of the different timescales of the two processes taking place in the system. The cooling of the meniscus is slow and lasts for minutes, see Fig. S8; in contrast, the drainage of the film from its initial thickness to the quasistationary one occurs over timescales of $\eta R_{\mathrm{d}} / \sigma_{0} \sim 10^{-5} \mathrm{~s}$ (S6). A similar situation was discussed by Marrucci (Marrucci, 1969) for his bubble coalescence problem, where the quasistationary foam film thickness is controlled by a slow diffusion process.

\section{Experimental observations of evaporating foam films}

The existing data on evaporating films from pure liquids (outlined in the Introduction) are scarce and contradictory, and do not allow the confirmation or rejection of the mechanism of stabilization proposed above. Therefore, we performed our own experimental tests of the predicted trends. For this purpose, we chose to investigate pure aqueous films under controlled moderate humidity $x$, as well as films formed from various pure alkanes. The evaporating alkane films are of practical relevance as a model for fuel and lubricant films occurring in the cylinders of internal combustion engines (Baumgarten, 2006). In addition, alkane films have certain advantages over aqueous from an experimental point of view, outlined below.

\subsection{Materials and methods}

We used a version of the Scheludko cell identical to the one described in (Kosior et al., 2014). For the convenience of the reader, its basic components are presented in Figure 1. Two capillaries of different radii $\left(R_{\mathrm{c}}=2\right.$ and $\left.2.3 \mathrm{~mm}\right)$ and wall thicknesses $\left(d_{\text {glass }} \sim 1\right.$ and $0.5 \mathrm{~mm}$, respectively) were used in order to test the reproducibility of the results. The capillaries were fixed inside a container of radius $\sim 1 \mathrm{~cm}$ and height $\sim 9 \mathrm{~cm}$, which was enclosed by a thermostat (HAAKE K10). The films were formed from a biconcave drop situated in the capillary (Figure 1A) by withdrawing liquid with a micropump (Hamilton). Observations of the films were made with an inverted optical microscope (Carl Zeiss Axiovert 200 MAT) connected to a camera (Hitachi HV-D20); the film thickness was determined interferometrically (Sheludko, 1967). Several dozens of films were formed for each loading of the apparatus; each run like this lasted for several hours. Runs were repeated up to three times to test the reproducibility. 
Aqueous films were formed from triply distilled water. For comparison, we tested also HPLC water, but the results were less reproducible. Following Manev (Manev, 1975), the humidity in the container was maintained (atmostated) by placing on the bottom of the sealed container a certain amount $(\sim 400 \mu \mathrm{L})$ of atmostating solution - concentrated $\mathrm{MgCl}_{2}$ in triply distilled water (Figure 1B). The solutions used were of concentration 1.60 and $4.93 \mathrm{~mol} / \mathrm{kg}$, corresponding to 89 and $45 \%$ relative humidity, respectively (Robinson and Stokes, 1959). Experiments under saturated conditions, with $\sim 400 \mu \mathrm{L}$ of pure water as the atmostating liquid, were also performed.

Hydrocarbon films were formed from hexane, nonane and hexadecane (Fluka), either with the container open to the ambient air (corresponding to zero vapour concentration, $x=0$ ), or with the container sealed and $600-800 \mu \mathrm{L}$ of the hydrocarbon placed on its bottom (maintaining saturated conditions, $x=1)$.

The observed films were normally circular and plane-parallel (Figure 1C), but with exceptions. We investigated films of different sizes - the experimental setup provides the opportunity to monitor the film radius with accuracy of $\pm 5 \mu \mathrm{m}$ and to control it manually with good precision. Once the film was formed, its radius remained constant during the whole drainage process until rupture, with the exception of the first several films in a run. The latter often expanded or shrunk significantly, perhaps in relation to highly undersaturated conditions; the atmostating liquid and the gas only equilibrate after several minutes. For each film, the rupture thickness $h_{\mathrm{cr}}$ and the film radius $R_{\mathrm{f}}$ were measured; for several of them, the whole $h(t)$ curve was recorded. Within a single run, the film stability exhibited a dependence on the time $t_{\text {load }}$ between the loading of the cell (the moment of the hermetic sealing of the container after the cell is loaded with fluid and the bottom of the container is loaded with atmostating liquid) and the formation of the film, so this parameter was also monitored. For films formed at similar $t_{\text {load, }}$ the values of $h_{\mathrm{cr}}$, the film lifetime $\tau$ and the whole $h(t)$ curve were reproducible within about $\pm 10 \%$.

Most atmostated film experiments were performed with the container sealed. Measurements were also performed with an open container and films exposed to the ambient humidity in the laboratory. This option was used as a qualitative test of the effect of abrupt changes in the humidity on the stability of the films. 
A number of experimental factors complicate the interpretation of the results obtained with the setup described above. One is the long time scale for reaching the stationary vapour pressure inside the container - it is of the order of $\sim L_{\text {cont }}^{2} / D \sim 5 \min \left(L_{\text {cont }}-\right.$ height of the container, $\sim 9$ $\mathrm{cm} ; D$-diffusion coefficient of water molecules in air, $2.6 \times 10^{-5} \mathrm{~m}^{2} / \mathrm{s}$ ). Due to that, the first $1-3$ films of each run drain under saturation between that in the laboratory (humidity 45-65\%) and the one set by the atmostating liquid. A related issue is that each film was formed in the cloud of vapour left after the previous one ruptured.

A second problem is the possible presence of surface-active contaminations, which can completely change the behaviour of the films we study. Even a very low concentration of surface-active impurities will cause a solutal Marangoni effect strong enough to counteract the thermal Marangoni effect discussed here. $\mathrm{Hu}$ and Larson ( $\mathrm{Hu}$ and Larson, 2005) have demonstrated that for an evaporating sessile drop, an adsorption as low as one molecule per 3000 $\mathrm{nm}^{2}$ can effectively suppress the Marangoni flow. In another paper (Hu and Larson, 2006), the same authors observed that the Marangoni flow pattern in an evaporating sessile droplet is accurately predicted by their theory for octane droplets, but for water, the experimental Marangoni flow is much weaker than expected, due to contaminants at the water surface. The impurities present in the alkane, on the other hand, are unlikely to have a significant affinity to the oil|air interface. To avoid contaminants, we used triply distilled water, and after each set of experiments, we lavishly washed the measuring cell and cleaned it in an oven at high temperature.

A third factor that introduces uncertainty into the interpretation of our experimental data on water films is the presence of $\mathrm{CO}_{2}$, whose dissolution in water introduces hydrogen carbonate and carbonate ions that allegedly charge the surface (Katsir and Marmur, 2014b) - this problem is also eliminated with the hydrocarbon films.

The validity of our model is restricted not only by these experimental complications, but also by our approximate treatment of the studied system, particularly the simplified model for the surface forces (29) and the approximate values of the numerical coefficients and $C_{p}$ in eqs. (19)(20)\&(23)-(25) (estimated for geometry of the system that only roughly matches the real one). Moreover, at high evaporation rates, non-linear effects related to convective transport and inertia are likely to be present. Therefore, we do not expect quantitative agreement between theory and experiment - we seek only qualitative correspondence, in terms of similar trends in response to 
variation of the experimental conditions and similar orders of magnitude of the relevant quantities $\left(h_{\mathrm{cr}}, \tau, R_{\mathrm{f}, \mathrm{cr}}\right.$ etc. $)$.

\subsection{Aqueous films}

In an undersaturated environment, the observed film lifetimes varied considerably with the time after cell loading $\left(t_{\text {load }}\right)$. Typically, each successive film would live shorter than the preceding one, provided that their radii were similar; this behaviour is illustrated in Figure 5A. In contrast, no significant changes with $t_{\text {load }}$ were observed under saturated conditions. The decrease of the lifetime between two consecutive films was more pronounced when the period between the formations of the two films was shorter, and when the films are formed at higher undersaturation. The films were also morphologically different depending on $t_{\text {load }}$ : at low loading times, films were not plane-parallel upon formation; at large $t_{\text {load, }}$ the films had approximately homogeneous thickness at their formation (Figure 6A\&B). The observed trend with $t_{\text {load }}$ can be explained with the fact that the formation of several consecutive evaporating films leads to a significant cooling of the glass holder. The meniscus, the glass capillary and probably the supporting glass tube are progressively colder at the formation of each next film and the time between the formations of two films is insufficient for them to relax to the ambient temperature $T_{\infty}$. If the initial temperature of the meniscus is lower than $T_{\infty}$, then the lifetime of the film will be decreased (see S8).

$\mathbf{1 0 0 \%}$ humidity. Stable, thick films were formed in saturated atmosphere (Figure 1C), in agreement with many previous reports (Exerowa, 1969; Yaminsky et al., 2010a) and contrary to what our model predicts. The films drained until reaching a plateau thickness of $h(t \rightarrow \infty)=60$ $110 \mathrm{~nm}$ and did not rupture on the timescale of the experiment. Even though the experiments lasted for several hours, there was no change of film stability with the passing of time after cell loading. By a small manual change of the pump pressure, the films could be forced to drain - a black spot formed and grew until at a certain point the film ruptured (Figure 6C). This behaviour is indicative of the existence of a weak barrier for the drainage.

The first two films formed right after the cell loading, while the air in the container was still not completely saturated, had a finite lifetime (50 and $90 \mathrm{~s}$ ), and their radii shrunk significantly (from 100 to $50 \mu \mathrm{m}$ ). 

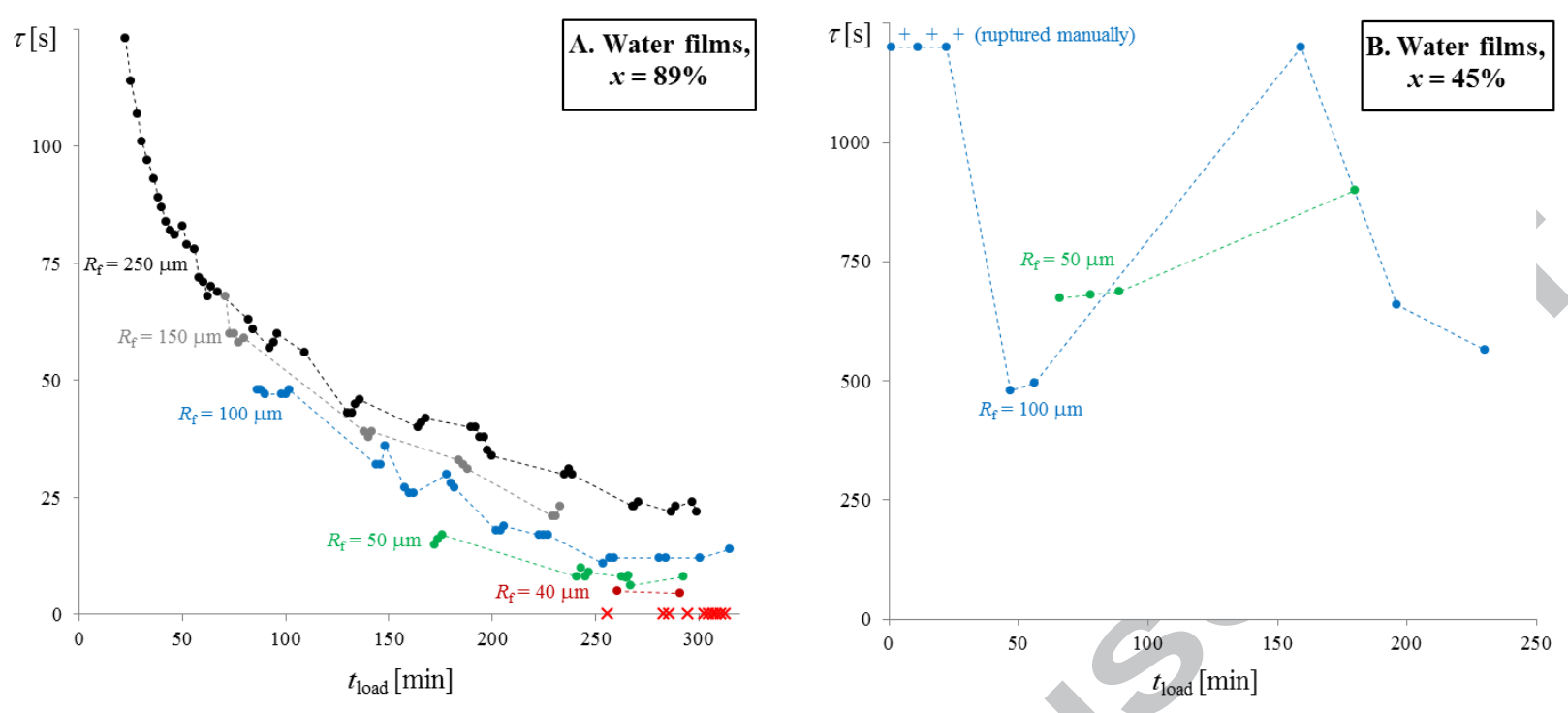

Figure 5. Dependence of the lifetime $\tau$ of water films during one run on the time $t_{\text {load }}$ after loading the cell. A. $89 \%$ humidity. Diagonal crosses indicate films that rupture instantly. B. $45 \%$ humidity. The first 3 films indicated with "+" were ruptured manually ten minutes after formation.
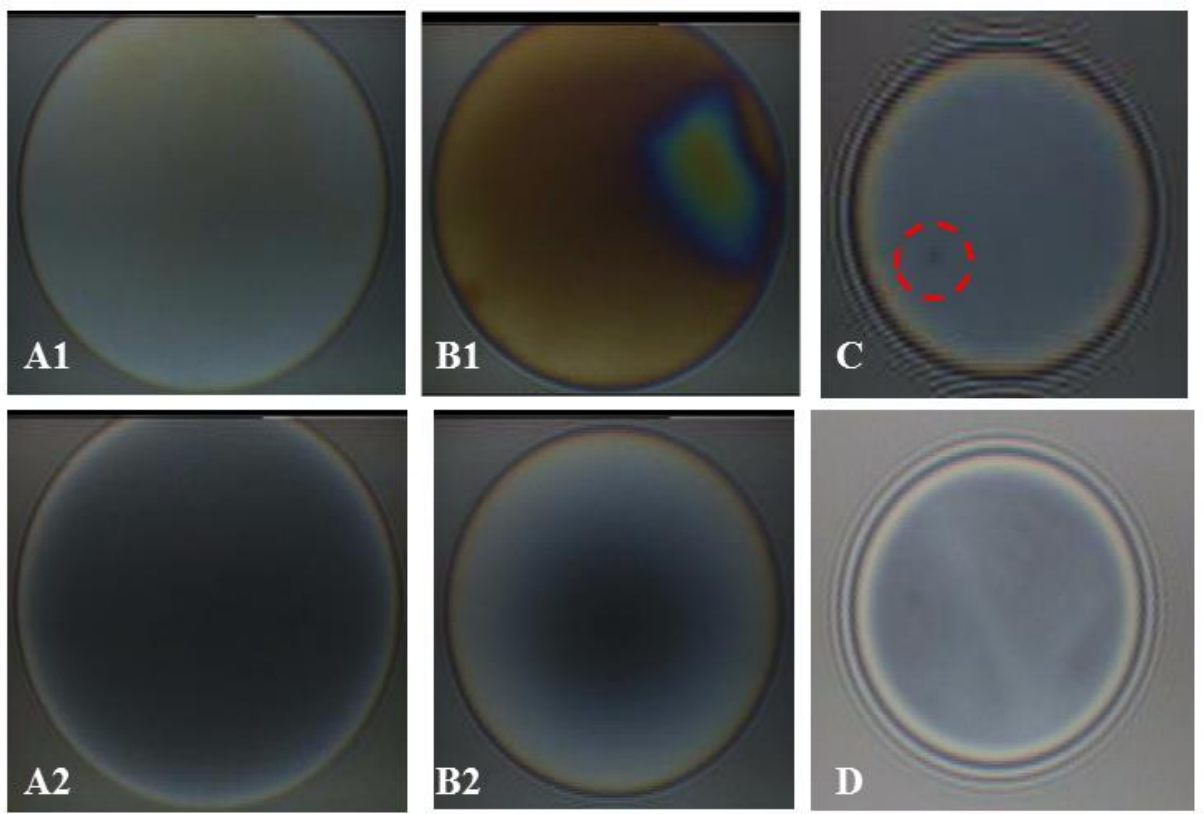

Figure 6. Representative images of the observed films. A. Water film, $x=89 \%$, high loading time, $R_{\mathrm{f}}=$ $250 \mu \mathrm{m}$; A1 - initial state, A2 - seconds before rupture. B. Water film, $x=89 \%$, low loading time, $R_{\mathrm{f}}=$ $250 \mu \mathrm{m}$; B1 - initial state, B2 - seconds before rupture. C. Water film, $x=100 \%$, black dot observed immediately before breakage. D. Nonane film, $x=100 \%, R_{\mathrm{f}} \sim 100 \mu \mathrm{m}$, lived for $50 \mathrm{~s}$. 
$89 \%$ humidity. Using eq. (40) with $\Delta T_{\mathrm{m}}=0$, we can calculate that the model predicts no stable films at $R_{\mathrm{f}}$ below $125 \mu \mathrm{m}$. However, we observed films of $R_{\mathrm{f}}=50-100 \mu \mathrm{m}$ exhibiting lifetimes of $10-50 \mathrm{~s}$, depending on $R_{\mathrm{f}}$ and $t_{\text {load }}$ (Figure $5 \mathrm{~A}$ ). For the films of radius $R_{\mathrm{f}}=150$ and $250 \mu \mathrm{m}$, the model predicts lifetimes of 37 and $145 \mathrm{~s}$, respectively. These values agree in order of magnitude with the experimentally measured range of 20-70 s for $R_{\mathrm{f}}=150 \mu \mathrm{m}$ and 20-300 s for $R_{\mathrm{f}}=250 \mu \mathrm{m}$ (the lifetimes still depend on $t_{\text {load }}$ ). The expected increase of the lifetime with the size of the films is confirmed - it is clearly seen in Figure $5 \mathrm{~A}\left(22.3 \pm 0.3^{\circ} \mathrm{C}, R_{\mathrm{c}}=2 \mathrm{~mm}\right)$. Large films lived longer at all loading times - for example, at $t_{\text {load }} \sim 180 \mathrm{~min}$, films of radius 40-200 $\mu \mathrm{m}$ drained for 5-35 s, respectively.

The films ruptured at a critical value of the thickness that varied in the range $h_{\mathrm{cr}}=26-48 \mathrm{~nm}$, in qualitative agreement with the value $40 \mathrm{~nm}$ predicted by our model, eq. (34). The observed $h_{\mathrm{cr}}$, however, depended on $t_{\text {load }}$ - the first few films ruptured at $26-30 \mathrm{~nm}$, while those at $t_{\text {load }}=250$ min ruptured at $40-48 \mathrm{~nm}$.

The very first film was extremely stable and lived for $5 \mathrm{~min}$ before we ruptured it manually - the most probable reason for its stability is that the container was not yet atmostated at the time when the film was formed $\left(t_{\text {load }}=17 \mathrm{~min}\right)$, i.e., $x$ was less than $89 \%$ and evaporation was more intense than it was for the following films.

Unstable films that ruptured immediately after formation were observed after $t_{\text {load }}=250 \mathrm{~min}$, and the frequency of their occurrence increased with $t_{\text {load }}$. At $t_{\text {load }}>300 \mathrm{~min}$, we could no longer observe films smaller than $R_{\mathrm{f}}=50 \mu \mathrm{m}$, which suggests that conditions at which $R_{\mathrm{f}, \mathrm{cr}}$ is above $50 \mu \mathrm{m}$ had been reached by that point.

45\% humidity. All films (formed at $22.0 \pm 0.3^{\circ} \mathrm{C}, R_{\mathrm{c}}=2.3 \mathrm{~mm}$ ) were very stable and lived much longer than the films at $x=89 \%$ - lifetimes were of the order of $10 \mathrm{~min}$, as illustrated in Figure 5B. This confirms qualitatively our theoretical prediction that lifetime increases with undersaturation, see Figure 4. The theoretical values of $\tau\left(\sim 140 \mathrm{~s}\right.$ for $R_{\mathrm{f}}=50 \mu \mathrm{m}, \sim 300 \mathrm{~s}$ for $R_{\mathrm{f}}=$ $100 \mu \mathrm{m})$ agree in order of magnitude with the experimental ones ( $\tau \sim 500-1000 \mathrm{~s})$. Let us remark that under such low humidities one of the approximations we used in our model fails: eq. (27) predicts that at $t \sim 500 \mathrm{~s}$, the meniscus temperature drops by $7 \mathrm{~K}$, and at such large temperature differences, the series expansion of the boundary condition (10) is inaccurate. In addition, these films were not plane parallel. 


\subsection{Hydrocarbon films}

n-Hexane films. Both for saturated conditions $(x=1)$ and for zero ambient concentration $(x=0)$, all films ruptured instantly. In contrast, at $x=0$, our theory predicts the existence of quasistationary hexane films with a lifetime of $\tau \sim 1000 \mathrm{~s}$ for $R_{\mathrm{f}}=200 \mu \mathrm{m}$. The discrepancy is likely due to hexane's high volatility (vapour pressure $19.9 \mathrm{kPa}$ ), which is beyond the approximations of our theoretical model. In particular, the convective mass transport, disregarded in our model, is not negligible for $\mathrm{C}_{6} \mathrm{H}_{14}$ - the mass transfer Péclet number, which measures the relative importance of convective diffusion, reaches values of $\sim 0.1$ for hexane (see S6).

n-Nonane. Saturated atmosphere, $x=1$. It can be expected that such films would break immediately in the absence of a repulsive disjoining pressure, but instead, they were stable for tens of seconds - an experimental run is illustrated in Figure 7. For more details on the behaviour of non-evaporating nonane films, see S9.

We employed different methods of perturbing the nonane films formed in a saturated environment. We first tried to perturb the liquid-vapour equilibrium by raising the temperature of the thermostat from 21 to $30^{\circ} \mathrm{C}$ ("cell heated" region in Figure 7). This was expected to result in a temporary undersaturation in the container which, according to the theoretical results in Section 2 , should in turn result in an increased stability of the film. Indeed, the first film formed after the temperature increase was very stable and lived for $210 \mathrm{~s}$. In the following minutes, the film lifetime decreased back to the values we observed for all non-evaporating nonane films (50-60 s), which suggests that by that time, the air in the container was already saturated with respect to the new temperature. We then changed the saturation more drastically by opening the container towards the air in the laboratory ("cell opened" region in Figure 7). For this case, we expect a gradient of the vapour pressure to arise inside the container, starting from complete saturation near the surface of the atmostating solution and reaching zero saturation at the container entrance. Assuming that the gradient is constant ( $C$ is a linear function of $z$ ) and that the meniscus is located at height of approximately $15 \%$ from the height $L_{\text {cont }}$ of the container, one can estimate that after the cell is opened, the relative saturation in the vicinity of the film is $\sim 85 \%$. Therefore, the opening of the container should increase the film lifetime. This was indeed 
observed: all films formed after the cell was opened lived for $\sim 140 \mathrm{~s}$, significantly more than those formed in a saturated environment.

Open cell, $x \approx 0$. Fifteen films of various sizes were formed in an open cell in the absence of atmostating liquid. Their behaviour was dependent on $t_{\text {load }}$ (Figure S10): the first five films expanded considerably after formation from $R_{\mathrm{f}}=50 \mu \mathrm{m}$ initially to $80-180 \mu \mathrm{m}$ just before rupture. The radius of all films formed 40 min after loading was stable, and $R_{\mathrm{f}}$ was either $\sim 50$, 100 or $150 \mu \mathrm{m}$. Their respective lifetimes were $\tau \sim 20,35$ and $45 \mathrm{~s}$; thus, in qualitative agreement with our theory (see Figure 4B), lifetime increased with $R_{\mathrm{f}}$. However, the experimental increase is relatively small compared to the theoretical: the model predicts that films with $R_{\mathrm{f}}=50 \mu \mathrm{m}$ do not reach a quasistationary state, and for $R_{\mathrm{f}}=100$ and $150 \mu \mathrm{m}$, the theoretical $\tau$ is 14 and $230 \mathrm{~s}$, respectively.

Interestingly, the films formed at $x=0$ were less stable than those formed at intermediate saturations $(x \approx 85 \%$ for films formed in the presence of atmostating liquid but in an open cell, see above), suggesting a non-monotonous dependence of the stability on $x$, in contrast with the predictions of our theory.

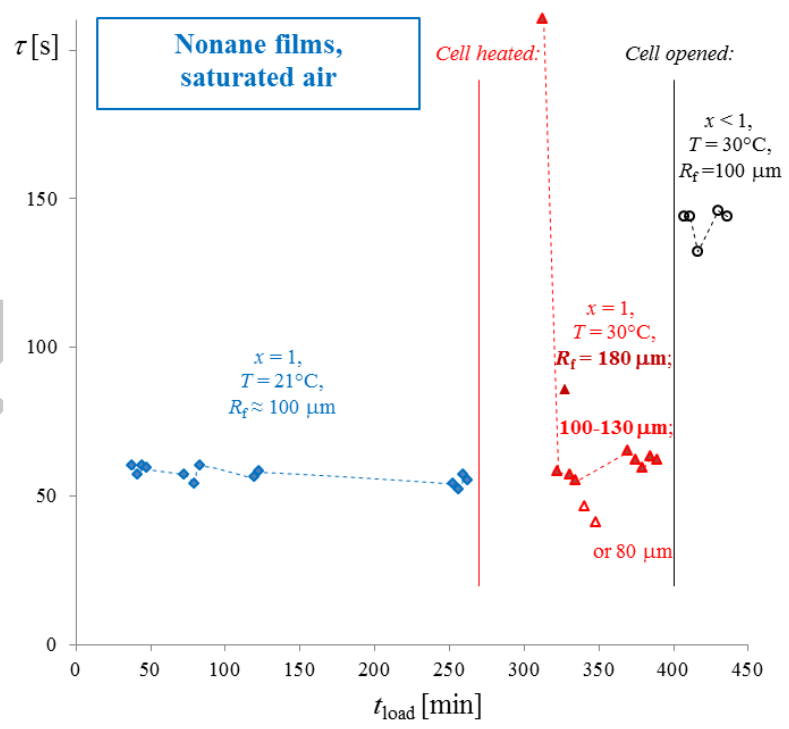

Figure 7. Lifetime $\tau$ of nonane films formed during one experimental run vs. time after loading the cell, $t_{\text {load }}\left(21^{\circ} \mathrm{C}, R_{\mathrm{c}}=2 \mathrm{~mm}\right.$, non-evaporating films in saturated environment). After $270 \mathrm{~min}$, the system was heated to $30^{\circ} \mathrm{C}$ - this resulted in a temporary decrease of the relative saturation and an increased stability of the film formed immediately afterwards. After $400 \mathrm{~min}$, the container was opened towards the air in the laboratory, which also decreased the saturation and increased $\tau$ of all films formed thereafter. 
The evolution of the film thickness $h(t)$ is compared to the theoretical one for a film of $R_{\mathrm{f}} \sim$ $125 \mu \mathrm{m}$ at $21^{\circ} \mathrm{C}$ in Fig. S11. The film drained from $\sim 260$ to $40 \mathrm{~nm}$, whereupon it ruptured. The theoretical $h(t)$ was calculated as explained in Section 2. Qualitative agreement between theory and experiment was observed, although the quantitative differences are significant. The critical thickness at which the films ruptured was $\sim 42.9 \mathrm{~nm}$ This is $30 \%$ less than theoretical value following from eq. (34), $60.3 \mathrm{~nm}$. The observed drainage rate is faster than the theoretical by a factor of about 5, which is reasonably good agreement in view of the major approximations employed in the calculation of the numerical coefficients in eqs. (19)-(20), (23)-(24) and (27).

n-Hexadecane. Experiments were performed under unsaturated conditions only $(x \sim 0)$. Instant rupture was observed in all cases. This is in agreement with the predictions of our theory: from eq. (38) it follows that the non-volatile hexadecane films are unstable over the whole experimental range of $R_{\mathrm{f}}$.

\section{Conclusions}

We have proposed a mechanism for the stabilization of evaporating foam films made of pure liquid, and gathered experimental evidence of its validity. Our theory explains the observed stability with the existence of a quasi-stationary state of the film sustained by the evaporationdriven thermal Marangoni flow. The evaporation of fluid causes a local temperature drop in the film region and a respective increase in the surface tension $\sigma$, which brings about a Marangoni flux from the meniscus toward the film. The predicted spatial variation of $\sigma$ across the film is relatively small $\left(\Delta \sigma \sim T_{r r} s^{\mathrm{S}} R_{\mathrm{f}}^{2} / 2 \sim 10^{-3}-10^{-2} \mathrm{mN} / \mathrm{m}\right.$ difference between the centre and the meniscus), but it is sufficient to stabilize a film formed from a pure liquid because its surfaces are tangentially mobile.

The experimentally observed decrease of the film thickness $h$ with time is explained with the evolution of the quasi-stationary state that follows the slow cooling down of the meniscus due to the evaporation (similar to Marrucci's theory (Marrucci, 1969), where the slow diffusion plays the same role). Once the meniscus reaches a certain critical temperature $T_{\mathrm{m}, \mathrm{cr}}$, the Marangoni effect can no longer counteract the attractive van der Waals force and the capillary pressure, leading to film rupture at a critical thickness $h_{\mathrm{cr}}$. The lifetime of the film is the time needed for the meniscus to cool down to $T_{\mathrm{m}, \mathrm{cr}}$. 
We were able to confirm experimentally several qualitative predictions of our theory, as follows.

(i) According to our model, films formed under lower ambient saturation, i.e., more intense evaporation, have an enhanced stability (Figure 4). This assertion is supported by a number of observations:

- the comparison between water films at $x=45 \%$ and $89 \%$ shows that lowering the humidity in the container indeed stabilizes the films - the lifetime is an order of magnitude longer at $x=$ $45 \%$, in agreement with the theoretical estimate;

- the comparison of the volatile nonane films vs. the non-volatile hexadecane films (both at $x$ $=0)$ shows that the evaporating nonane films are quite stable, while the non-evaporating hexadecane films rupture upon formation;

- perturbing the liquid-vapour equilibrium by raising the temperature or by opening the container leads to faster evaporation and more stable nonane films, Figure 7;

- the increased stability of the first film formed during each run in the case of water films at $x=89 \%$ can be explained with the air in the container still being undersaturated (the complete saturation requires several minutes);

- the expected trend of $\tau$ vs. $x$. has been observed by other authors - according to Karakashev and Firouzi (Karakashev and Firouzi, 2014), pure water films break upon formation at $97 \%$ humidity and live for several seconds at $x=78-88 \%$.

(ii) The model predicts that films of greater radii have longer lifetimes, see Figure 4B, which is confirmed for most evaporating water and nonane films, Figure 5 and Figure S10. The experimental and the theoretical trends of the dependence $\tau$ vs. $R_{\mathrm{f}}$ are in adequate agreement. The theory predicts that films of size smaller than a certain critical value $R_{\mathrm{f}, \mathrm{cr}}$ must be unstable. With water films at $89 \%$, we found some evidence for the existence of $R_{\mathrm{f}, \mathrm{cr}}-$ under these conditions we observed many unstable films that rupture immediately upon formation and no films with a radius of less than $100 \mu \mathrm{m}$ were stable after $t_{\text {load }}=300 \mathrm{~min}$, see Figure $5 \mathrm{~A}$.

(iii) The model predicts the existence of a critical thickness $h_{\mathrm{cr}}$ of film rupture, at which the thermal Marangoni mechanism of stabilization ceases to act, eq. (34). The experimentally measured $h_{\text {cr }}$ of water and nonane compare adequately with the theoretical value. The model also gives an approximate expression for the evolution of the film thickness with time which is in 
qualitative agreement with the experimental one (although the observed rate of thinning is faster than the theoretical, Fig. S11).

Some of these observations can be explained with other hypothetical stabilizing mechanisms, but not the combination of them. For example, the assumption for the existence of electrostatic repulsion can lead to a critical thickness of rupture similar to that in the ScheludkoVrij (Radoev et al., 1983; Vrij, 1966) mechanism of film rupture through the occurrence of capillary waves; it, however, cannot explain the effect of the abrupt changes of the saturation on the film stability, nor the trend of the dependence of the lifetime $\tau$ on the film radius $R_{\mathrm{f}}$. The presence of impurities (Yaminsky et al., 2010a) and dissolved and dissociated $\mathrm{CO}_{2}$ (Katsir and Marmur, 2014b) may in part explain the behaviour of water films, but not that of alkane films. We can therefore say that the phenomena summarized in (i-iii) above have their simplest possible explanation in the evaporation-driven thermal Marangoni effect.

Yet, it must not be overlooked that several of our observations do not align with the theoretical predictions. Nearly all of these points of disagreement could be traced to the unanswered question of why pure non-evaporating films are stable. We obtained the following experimental results that give useful hints about the nature of this stabilization:

(i) We consistently obtained aqueous films that were stable in a saturated atmosphere, thus confirming the results of Exerowa (Exerowa, 1969) and Yaminsky et al. (Yaminsky et al., 2010a). These films drained until they reached a stable thickness $\sim 100 \mathrm{~nm}$ and had a lifetime longer than $30 \mathrm{~min}$. This stability is indicative of the presence of an additional force, probably of electrostatic nature (Yaminsky et al., 2010b).

(ii) We consistently obtained non-evaporating nonane films of relatively high stability (of the order of tens of seconds). In contrast, our hexane films and Yaminsky's (Yaminsky, 2006) npentane films were unstable in a saturated atmosphere. It is puzzling that films from short- and long-chained alkanes behave differently in saturated atmosphere; it is also puzzling that water films are stable for hours while nonane films break after a minute. With nonane films, there is a clear dependence of film lifetime on film radius, i.e., larger non-evaporating films live longer.

(iii) We observed that non-evaporating films are typically more stable than slowly evaporating films. Water films formed after the complete saturation of the container $(x=100 \%$, $t_{\text {load }}>9 \mathrm{~min}$ ) had indefinitely long lifetimes, while the first films formed before saturation lived for $40-50 \mathrm{~s}$, and films at $x=89 \%$ humidity lived for about a minute. Introducing a low rate of 
evaporation also noticeably destabilized the nonane films. These experimental findings contradict our theory. Another discrepancy is that our model predicts no stable films at humidities above a certain critical value $x_{\text {cro }}$ (eq. (38) and Figure 4), which is not confirmed by the data for water and nonane.

These discrepancies are not surprising in view of the fact that our model neglects the electrostatic repulsion under all circumstances and therefore incorrectly predicts that pure nonevaporating films should rupture immediately (compare with S7). However, devising an accurate model of the electrostatic disjoining pressure in a pure liquid is a challenging task that goes well beyond the aims of our current study. The double layer in pure water films is of a very sparse structure - the concentration of ions corresponds to one ion per $300 \times 300 \mathrm{~nm}^{2}$, compared to a film thickness of $100 \mathrm{~nm}$. The characteristic distances of the electrostatic interaction are the Debye length and the average distance between two ions - for pure water, both are of the order of hundreds of nanometres, more than the film thickness. Electric double layers of such sparse structure have not, to our knowledge, been investigated, and may give rise to various peculiar effects - for example, the time required for the formation of the sparse double layer is of the order of $L_{\mathrm{D}}^{2} / D_{\text {ion }} \sim 0.1 \mathrm{~ms}$ for water, and much more for alkanes (Klinkenberg and Minne, 1958). A fast dynamic phenomenon such as the formation of a foam film will be able to perturb this structure (by analogy with the effect of turbulence on electrokinetic phenomena in oils (Klinkenberg and Minne, 1958)) and perhaps decrease the repulsion. In view of these comments, our tentative hypothesis about the observed discrepancies between theory and experiment is that low evaporation rates decrease film stability due to an unclear mechanism of perturbation of the structure of the sparse electric double layer in the film, leading to suppression of the electrostatic repulsion that stabilizes the non-evaporating films. Further increase of the evaporation leads to stabilization, in accordance with our theory.

The study presented here is a first step towards the explanation of a family of similar phenomena, such as: the stability of evaporating films formed from concentrated electrolyte solutions (Karakashev et al., 2008); the destabilization of evaporating films by small amounts of non-volatile surfactants (Yaminsky et al., 2010a); the stability of evaporating films containing volatile surface-active agents (e.g., the dynamic stabilization of evaporating films formed from aqueous butanol solutions indicated by an unpublished experimental study of ours). Naturally, additional complications are expected in these systems because all of them involve conjugated 
thermal and solutal Marangoni effects. Phenomena of practical importance such as the occurrence of reflux in the injector nozzle after the injection event (Wang, 2012) are probably driven by a combination of such effects.

Acknowledgements. R.S. acknowledges support from BP through the BP International Centre for Advanced Materials (BP-ICAM). J.N. thanks Trinity College and the Clarendon Fund of the University of Oxford for a Clarendon-Titley Scholarship. Numerous discussions with Stoyan Karakashev, Emil Manev, Boryan Radoev, Roumen Tsekov, Krassimir Danov, Sorin Filip and Colin Bain helped us with the formulation of the problem.

\section{Supplementary information:}

S1. List of symbols from the main text and values of the parameters

S2. Hydrodynamics

S3. Heat currents through the liquid and the air

S4. Mass current through the air

S5. Knudsen and Fuchs transition layers

S6. Properties of the system at the stationary state for a thermostated meniscus

S7. Effect of the electrostatic disjoining pressure

S8. The cooling of the meniscus

S9. Additional experimental data

\section{References}

Baumgarten, C., 2006. Mixture Formation in Internal Combustion Engine, Heat, Mass Transfer. Springer Berlin Heidelberg, Berlin. doi:10.1007/3-540-30836-9

Boinovich, L., 2010. DLVO forces in thin liquid films beyond the conventional DLVO theory. Curr. Opin. Colloid Interface Sci. 15, 297-302. doi:10.1016/j.cocis.2010.05.003

Chandramohan, A., Dash, S., Weibel, J.A., Chen, X., Garimella, S. V, 2016. Marangoni Convection in Evaporating Organic Liquid Droplets on a Nonwetting Substrate. Langmuir 32, 4729-4735. doi:10.1021/acs.langmuir.6b00307

Danov, K.D., Alleborn, N., Raszillier, H., Durst, F., 1998. The stability of evaporating thin liquid films in the presence of surfactant. I. Lubrication approximation and linear analysis. Phys. 
Fluids 10, 131-143. doi:10.1063/1.869555

Del Castillo, L.A., Ohnishi, S., Horn, R.G., 2011. Inhibition of bubble coalescence: Effects of salt concentration and speed of approach. J. Colloid Interface Sci. 356, 316-324. doi:10.1016/j.jcis.2010.12.057

Derjaguin, B.V., 1986. Theory of the Stability of Colloids and Thin Films (in Russian). Nauka, Moscow.

Dombrovsky, L.A., Sazhin, S.S., 2003. A simplified non-isothermal model for droplet heating and evaporation. Int. Commun. Heat Mass Transf. 30, 787-796. doi:10.1016/S07351933(03)00126-X

Du, L., Folliard, K.J., 2005. Mechanisms of air entrainment in concrete. Cem. Concr. Res. 35, 1463-1471. doi:10.1016/j.cemconres.2004.07.026

Exerowa, D., 1969. Effect of adsorption, ionic strength and $\mathrm{pH}$ on the potential of the diffuse electric layer. Kolloid-Zeitschrift und Zeitschrift für Polym. 232, 703-710. doi:10.1007/BF01500168

Exerowa, D., Kruglyakov, P.M., 1998. Foam and Foam Films. Theory, Experiment, Application. Elsevier, Amsterdam.

Firouzi, M., Nguyen, A. V., 2014. Effects of monovalent anions and cations on drainage and lifetime of foam films at different interface approach speeds. Adv. Powder Technol. 25, 1212-1219. doi:10.1016/j.apt.2014.06.004

Fuchs, N.A., 1959. Evaporation and Droplet Growth in Gaseous Media. Pergamon Press, London.

Hu, H., Larson, R.G., 2006. Marangoni Effect Reverses Coffee-Ring Depositions. J. Phys. Chem. B 110, 7090-7094. doi:10.1021/jp0609232

Hu, H., Larson, R.G., 2005. Analysis of the Effects of Marangoni Stresses on the Microflow in an Evaporating Sessile Droplet. Langmuir 21, 3972-3980. doi:10.1021/la0475270

Israelachvili, J.N., 2011. Intermolecular and Surface Forces, 3rd ed. Academic Press, London.

Ivanov, I.B., Dimitrov, D.S., 1974. Hydrodynamics of thin liquid films. Colloid Polym. Sci. 252, 982-990. doi:10.1007/BF01566619

Karakashev, S.I., Firouzi, M., 2014. Personal Communication.

Karakashev, S.I., Nguyen, P.T., Tsekov, R., Hampton, M.A., Nguyen, A. V., 2008. Anomalous Ion Effects on Rupture and Lifetime of Aqueous Foam Films Formed from Monovalent Salt 
Solutions up to Saturation Concentration. Langmuir 24, 11587-11591. doi:10.1021/la801456j

Katsir, Y., Marmur, A., 2014a. Rate of Bubble Coalescence Following Dynamic Approach: Collectivity-Induced Specificity of Ionic Effect. Langmuir 30, 13823-13830. doi:10.1021/la503373d

Katsir, Y., Marmur, A., 2014b. Rate of Bubble Coalescence following Quasi-Static Approach: Screening and Neutralization of the Electric Double Layer. Sci. Rep. 4, 4266. doi:10.1038/srep04266

Klinkenberg, A., Minne, J.L. van der (Eds.), 1958. Electrostatics in the Petroleum Industry: The Prevention of Explosion Hazards. A Royal Dutch-Shell Research and Development Report. Elsevier, Amsterdam.

Kolarov, T., Yankov, R., Esipova, N.E., Exerowa, D., Zorin, Z.M., 1993. Charge reversal at the air/water interface as inferred from the thickness of foam films. Colloid Polym. Sci. 271, 519-520. doi:10.1007/BF00657398

Kosior, D., Zawala, J., Todorov, R., Exerowa, D., Malysa, K., 2014. Bubble bouncing and stability of liquid films formed under dynamic and static conditions from n-octanol solutions. Colloids Surf., A. 460,391-400. doi:10.1016/j.colsurfa.2013.11.022

Levich, V.G., 1962. Physicochemical Hydrodynamics. Prentice-Hall, Inc., Englewood Cliffs, N.J.

Li, X., Karakashev, S.I., Evans, G.M., Stevenson, P., 2012. Effect of Environmental Humidity on Static Foam Stability. Langmuir 28, 4060-4068. doi:10.1021/la205101d

Manev, E., Tsekov, R., Radoev, B., 1997. Effect of thickness non-homogeneity on the kinetic behaviour of microscopic foam film. J. Dispers. Sci. Technol. 18, 769-788. doi:10.1080/01932699708943771

Manev, E.D., 2013. Personal Communication.

Manev, E.D., 1975. Effect of the disjoining pressure and surfactant diffusion on the thinning rate of aniline foam films. Ann. Univ. Sofia Fac. Chem. 70, 97-109.

Manev, E.D., Nguyen, A. V., 2005. Effects of surfactant adsorption and surface forces on thinning and rupture of foam liquid films. Int. J. Miner. Process. 77, 1-45. doi:10.1016/j.minpro.2005.01.003

Manica, R., Chan, D.Y.C., 2011. Drainage of the air-water-quartz film: experiments and theory. 
Phys. Chem. Chem. Phys. 13, 1434-1439. doi:10.1039/C0CP00677G

Marinova, K.G., Alargova, R.G., Denkov, N.D., Velev, O.D., Petsev, D.N., Ivanov, I.B., Borwankar, R.P., 1996. Charging of Oil-Water Interfaces Due to Spontaneous Adsorption of Hydroxyl Ions. Langmuir 12, 2045-2051. doi:10.1021/la950928i

Marrucci, G., 1969. A theory of coalescence. Chem. Eng. Sci. 24, 975-985. doi:10.1016/00092509(69)87006-5

Nguyen, P.T., Nguyen, A. V, 2010. Drainage, Rupture, and Lifetime of Deionized Water Films: Effect of Dissolved Gases? Langmuir 26, 3356-3363. doi:10.1021/la9031333

Oron, A., Davis, S.H., Bankoff, S.G., 1997. Long-scale evolution of thin liquid films. Rev. Mod. Phys. 69, 931-980. doi:10.1103/RevModPhys.69.931

Palit, S.R., 1956. Thermodynamic Interpretation of the Eötvös Constant. Nature 177, 1180-1180. doi:10.1038/1771180a0

Peng, T., Chang, T.-M., 2014. Molecular processes of ion effects on aqueous nanofilm rupture. J. Mol. Liq. 193, 139-151. doi:10.1016/j.molliq.2013.12.031

Radoëv, B.P., Dimitrov, D.S., Ivanov, I.B., 1974. Hydrodynamics of thin liquid films effect of the surfactant on the rate of thinning. Colloid Polym. Sci. 252, 50-55. doi:10.1007/BF01381695

Radoev, B.P., Scheludko, A.D., Manev, E.D., 1983. Critical thickness of thin liquid films: Theory and experiment. J. Colloid Interface Sci. 95, 254-265. doi:10.1016/00219797(83)90094-2

Reynolds, O., 1886. On the Theory of Lubrication and Its Application to Mr. Beauchamp Tower's Experiments, Including an Experimental Determination of the Viscosity of Olive Oil. Proc. R. Soc. London 40, 191-203. doi:10.1098/rspl.1886.0021

Robinson, R.A., Stokes, R.H., 1959. Electrolyte Solutions, 2nd ed. Butterworths Scientific Publications, London.

Scheludko, A., Platikanov, D., Manev, E., 1965. Disjoining pressure in thin liquid films and the electro-magnetic retardation effect of the molecule dispersion interactions. Discuss. Faraday Soc. 40, 253. doi:10.1039/df9654000253

Sheludko, A., 1967. Thin liquid films. Adv. Colloid Interface Sci. 1, 391-464. doi:10.1016/00018686(67)85001-2

Slavchov, R.I., Karakashev, S.I., Ivanov, I.B., 2014. Ionic Surfactants and Ion-Specific Effects: 
Adsorption, Micellization, and Thin Liquid Films, in: Romsted, L.S. (Ed.), Surfactant Science and Technology: Retrospects and Prospects. CRC Press, Boca Raton, FL, pp. 53117.

Slavchov, R.I., Novev, J.K., 2012. Surface tension of concentrated electrolyte solutions. J. Colloid Interface Sci. 387, 234-243. doi:10.1016/j.jcis.2012.07.020

Snegirev, A.Y., 2013. Transient temperature gradient in a single-component vaporizing droplet. Int. J. Heat Mass Transf. 65, 80-94. doi:10.1016/j.ijheatmasstransfer.2013.05.064

Stubenrauch, C., Klitzing, R. von, 2003. Disjoining pressure in thin liquid foam and emulsion films - new concepts and perspectives. J. Phys. Condens. Matter 15, R1197-R1232. doi:10.1088/0953-8984/15/27/201

Sullivan, A.P., Kilpatrick, P.K., 2002. The Effects of Inorganic Solid Particles on Water and Crude Oil Emulsion Stability. Ind. Eng. Chem. Res. 41, 3389-3404. doi:10.1021/ie010927n

Sultan, E., Boudaoud, A., Amar, M. Ben, 2005. Evaporation of a thin film: diffusion of the vapour and Marangoni instabilities. J. Fluid Mech. 543, 183. doi:10.1017/S0022112005006348

Torrie, G.M., Valleau, J.P., Outhwaite, C.W., 1984. Electrical double layers. VI. Image effects for divalent ions. J. Chem. Phys. 81, 6296-6300. doi:10.1063/1.447536

Tsekov, R., Ruckenstein, E., 1994. Dimple formation and its effect on the rate of drainage in thin liquid films. Colloids Surf., A. 82, 255-261. doi:10.1016/0927-7757(93)02633-P

Tsoumpas, Y., Dehaeck, S., Rednikov, A., Colinet, P., 2015. Effect of Marangoni Flows on the Shape of Thin Sessile Droplets Evaporating into Air. Langmuir 31, 13334-13340. doi:10.1021/acs.langmuir.5b02673

Valkovska, D.S., Danov, K.D., 2001. Influence of Ionic Surfactants on the Drainage Velocity of Thin Liquid Films. J. Colloid Interface Sci. 241, 400-412. doi:10.1006/jcis.2001.7757

Vrij, A., 1966. Possible mechanism for the spontaneous rupture of thin, free liquid films. Discuss. Faraday Soc. 42, 23. doi:10.1039/df9664200023

Wang, L., Qu, X., 2012. Impact of interface approach velocity on bubble coalescence. Miner. Eng. 26, 50-56. doi:10.1016/j.mineng.2011.10.016

Wang, Y.C., 2012. Study of Deposit Formation Inside Diesel Injectors Nozzles. PhD Thesis. Massachusetts Institute of Technology.

Yaminsky, V.V., 2006. Bubble vortex at surfaces of evaporating liquids. J. Colloid Interface Sci. 
297, 251-260. doi:10.1016/j.jcis.2005.10.004

Yaminsky, V. V., Ohnishi, S., Vogler, E.A., Horn, R.G., 2010a. Stability of Aqueous Films between Bubbles. Part 2. Effects of Trace Impurities and Evaporation. Langmuir 26, 80758080. doi:10.1021/la904482n

Yaminsky, V. V., Ohnishi, S., Vogler, E.A., Horn, R.G., 2010b. Stability of Aqueous Films between Bubbles. Part 1. The Effect of Speed on Bubble Coalescence in Purified Water and Simple Electrolyte Solutions. Langmuir 26, 8061-8074. doi:10.1021/la904481d

Yiantsios, S.G., Higgins, B.G., 2006. Marangoni flows during drying of colloidal films. Phys. Fluids 18, 82103. doi:10.1063/1.2336262

Zuiderweg, F.J., Harmens, A., 1958. The influence of surface phenomena on the performance of distillation columns. Chem. Eng. Sci. 9, 89-103. doi:10.1016/0009-2509(58)80001-9 


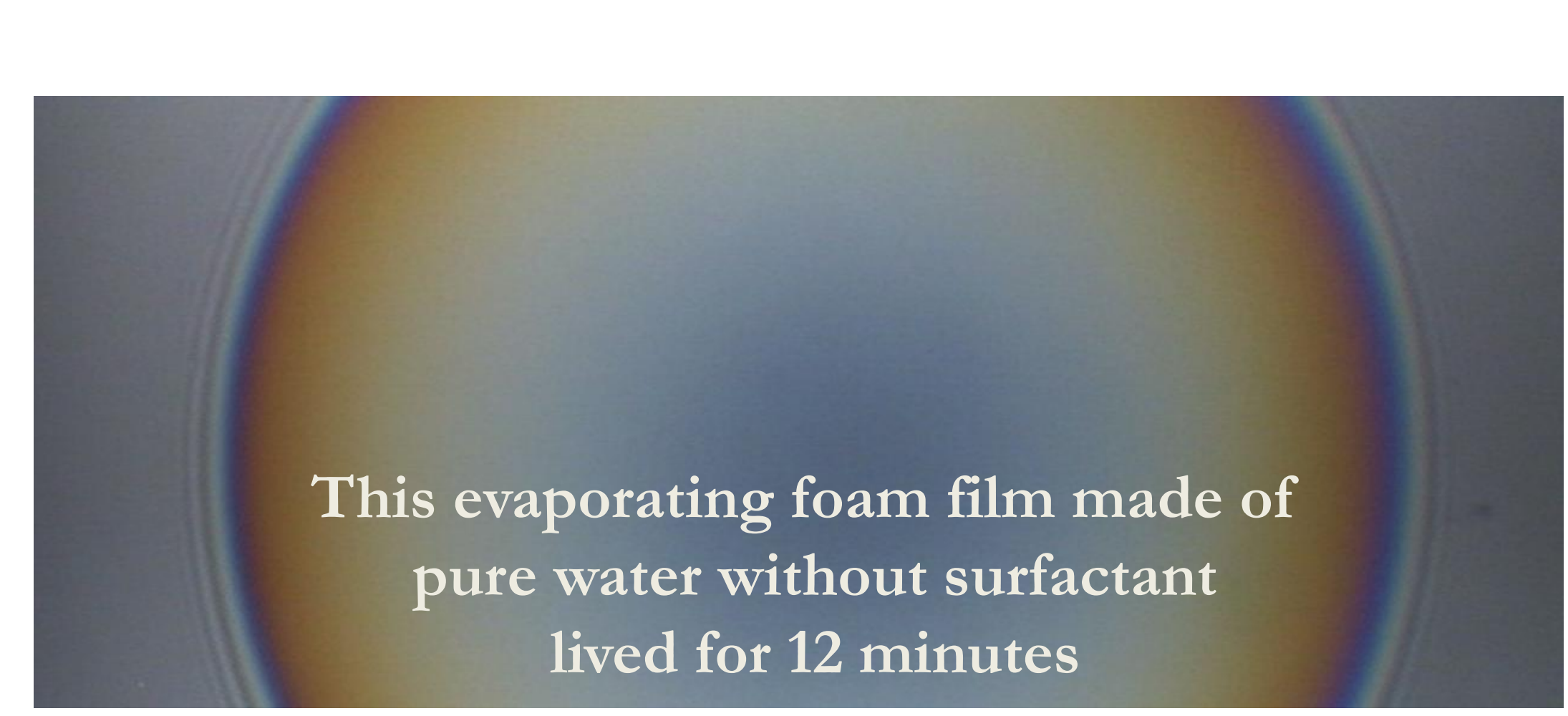

$$
\text { t }
$$

\footnotetext{
tact
}

\section{Graphical Abstract}

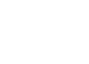
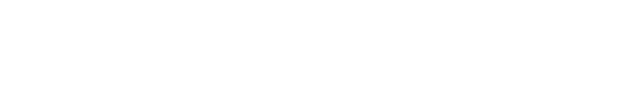

\section{This evaporating foam film made of pure water without surfactant lived for 12 minutes} (
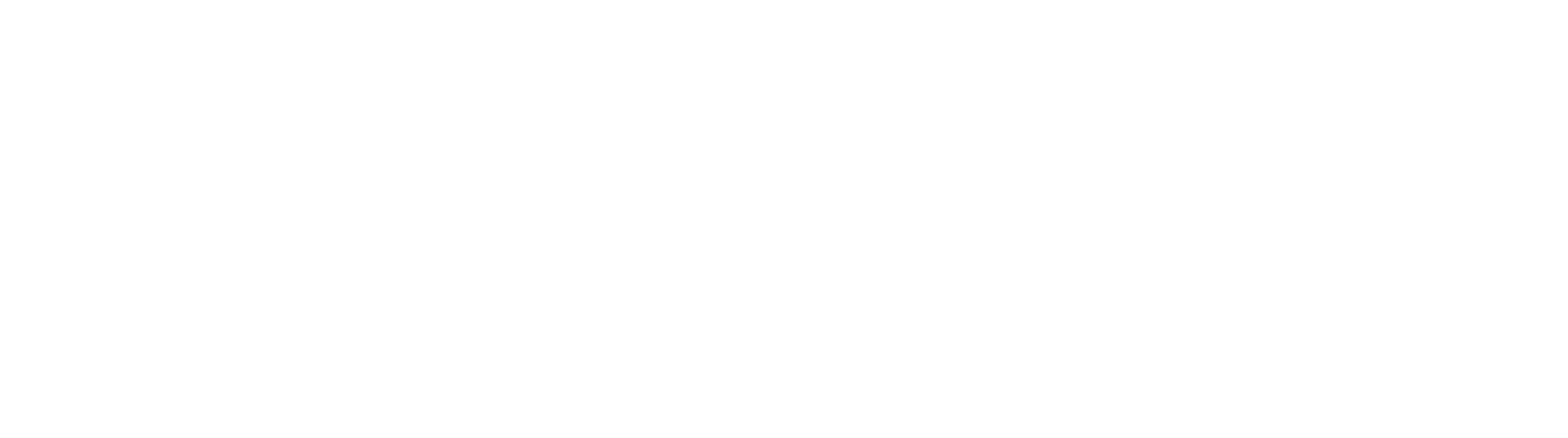

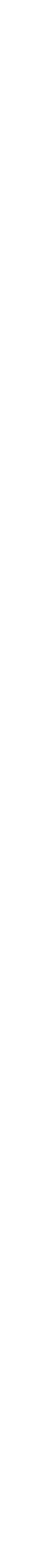




\section{Highlighs:}

Evaporation stabilizes foam films made of pure liquids.

Evaporation induces temperature difference between the film and the meniscus.

The temperature gradient induces a stabilizing thermal Marangoni flow.

The Marangoni flow sustains a stationary thickness of the foam film.

The film thins as the meniscus cools down, until rupture at a critical thickness. 